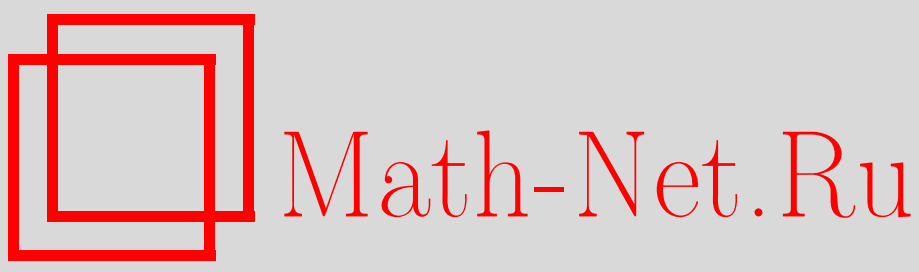

Вик. С. Куликов, М. Тайхер, Брэйд-монодромные разложения и диффеоморфные типы, Изв. РАН. Сер. матем., 2000, том 64, выпуск 2, 89-120

DOI: https://doi.org/10.4213/im285

Использование Общероссийского математического портала Math-Net.Ru подразумевает, что вы прочитали и согласны с пользовательским соглашением http://www . mathnet.ru/rus/agreement

Параметры загрузки:

IP: 54.198 .64 .247

26 апреля 2023 г., 13:42:33 
УДК $512.7+515.1$

Вик. С. Куликов, М. Тайхер

\title{
Брэйд-монодромные разложения и диффеоморфные типы
}

\begin{abstract}
В статье доказывается, что если две плоские каспидальные кривые $B_{1}$ и $B_{2}$ имеют эквивалентные брэйд-монодромные разложения на множители, то кривые $B_{1}$ и $B_{2}$ гладко изотопны в $\mathbb{C P}^{2}$. В качестве следствия получаем, что если дискриминантные кривые (кривые ветвления) $B_{1}$ и $B_{2}$ общих проекций на $\mathbb{C P}^{2}$ поверхностей общего типа $S_{1}$ и $S_{2}$, вложенных в проективное пространство с помощью кратного канонического класса, имеют эквивалентные брэйд-монодромные разложения на множители, то $S_{1}$ и $S_{2}$ (рассматриваемые как вещественные четырехмерные многообразия) являются диффеоморфными.

Библиография: 8 наименований
\end{abstract}

\section{Введение}

Пусть $S \subset \mathbb{C P}^{r}$ - неособая проективная алгебраическая поверхность степени $\operatorname{deg} S=N$. Хорошо известно, что для почти всех проекций $p r: \mathbb{C P}^{r} \rightarrow \mathbb{C P}^{2}$ ограничения $f: S \rightarrow \mathbb{C P}^{2}$ этих проекций на $S$ удовлетворяют следующим условиям:

i) $f$ является конечным морфизмом степени $\operatorname{deg} f=\operatorname{deg} S$;

ii) $f$ разветвлен вдоль неприводимой кривой $B \subset \mathbb{C P}^{2}$, особые точки которой суть лишь обыкновенные каспы и ноуды;

iii) $f^{*}(B)=2 R+C$, где кривая $R$ неприводима и неособа, а $C$ приведена;

iv) морфизм $\left.f\right|_{R}: R \rightarrow B$ совпадает с морфизмом нормализации кривой $B$.

Мы будем называть такой морфизм $f$ общим морфизмом, а его кривую ветвления $B$ - дискриминантной кривой морфизма $f$.

Скажем, что два общих морфизма $\left(S_{1}, f_{1}\right),\left(S_{2}, f_{2}\right)$ с одной и той же дискриминантной кривой $B$ являются эквивалентными, если существует изоморфизм $\varphi: S_{1} \rightarrow S_{2}$ такой, что $f_{1}=f_{2} \circ \varphi$.

Следующее утверждение известно как гипотеза Кизини (Chisini).

ГИПОТЕЗА КИЗИНИ. Пусть $B$ - дискриминантная кривая общего морфизма $f: S \rightarrow \mathbb{C P}^{2}$ степени $\operatorname{deg} f \geqslant 5$. Тогда $f$ однозначно определяется парой $\left(\mathbb{C P}^{2}, B\right)$.

Легко видеть, что аналогичное утверждение для общих морфизмов проективных кривых на $\mathbb{C P}^{1}$ неверно. С другой стороны, в [3] было показано, что гипотеза Кизини выполнена для дискриминантных кривых почти всех общих морфизмов произвольной проективной поверхности. В частности, если $S$ - произвольная поверхность общего типа с обильным каноническим классом, то гипотеза Кизини выполнена для дискриминантных кривых общих морфизмов $f: S \rightarrow \mathbb{C P}^{2}$, заданных

Работа выполнена при частичной финансовой поддержке грантов INTAS-OPEN-97-2072, РФФИ № 99-01-01133, а также поддержана институтом математики Макса Планка (Бонн, Германия), Исследовательским институтом математики им. Эмми Нётер, Университетом Бар-Илан и фондом Минерва.

(C) Вик.С. КУликов, М. ТАйхеР, 2000 
трехмерной линейной подсистемой из $m$-канонического класса поверхности $S$, где $m \in \mathbb{N}$. Дискриминантные кривые таких обших морфизмов мы будем называть т-каноническими дискриминантнылми кривыми.

Пусть $B$ - алгебраическая кривая в $\mathbb{C P}^{2}$ степени $p$. Топология вложения $B \subset$ $\mathbb{C P}^{2}$ определяется брәйд-монодромией кривой $B$, которая описьвается разложением “полного оборота" $\Delta_{p}^{2}$ в полугруппе $B_{p}^{+}$группы кос $B_{p}$ из $p$ нитей $\left(\Delta_{p}^{2}=\right.$ $\left(X_{1} \cdot \ldots \cdot X_{p-1}\right)^{p}$ в стандартных образуюших группы кос). Если $B$ - каспидальная кривая, то это разложение может быть записано в виде

$$
\Delta_{p}^{2}=\prod_{i} Q_{i}^{-1} X_{1}^{\rho_{i}} Q_{i}, \quad \rho_{i} \in(1,2,3)
$$

где $X_{1}$ - положительный полуповорот в $B_{p}$.

Пусть

$$
h=g_{1} \cdot \ldots \cdot g_{r}
$$

- разложение в $B_{p}^{+}$. Преобразование, которое меняет местами два соседних множителя в $(2)$ по правилу $g_{i} \cdot g_{i+1} \longmapsto\left(g_{i} g_{i+1} g_{i}^{-1}\right) \cdot g_{i}$, либо $g_{i} \cdot g_{i+1} \longmapsto g_{i+1}\left(g_{i+1}^{-1} g_{i} g_{i+1}\right)$, называется заменой Гурвица.

Для $z \in B_{p}$ обозначим $h_{z}=z^{-1} g_{1} z \cdot z^{-1} g_{2} z \cdot \ldots \cdot z^{-1} g_{r} z$ и скажем, что разложение $h_{z}$ получено из (2) одновременным сопряжением на $z$. Два разложения называются әквивалентными по отношению замен Гурвица и сопряжения, если одно из них может быть получено из другого в процессе конечного числа замен Гурвица с последуюшим одновременным сопряжением на некоторьй элемент $z \in B_{p}$. Мы скажем, что два разложения вида (1) принадлежат одному и тому же muпy брәйд-разложения, если они эквивалентны по отношению замен Гурвица и сопряжения. Основными вопросами в данном направлении являются следующие проблемы.

ПроБлемА 1. Пусть $B \subset \mathbb{C P}^{2}$ - каспидальная кривая. Определяет ли однозначно тип брәйд-разложсения пары $\left(\mathbb{C P}^{2}, B\right)$ диффеоморфный тип этой парье и обратно?

ПрОБЛЕМА 2. Пусть $\Delta_{p}^{2}=\mathscr{E}_{1} u \Delta_{p}^{2}=\mathscr{E}_{2}-$ два брәйд-монодромных разложения на множители. Существует ли конечный алгоритм распознавания: принадлежат или нет эти два брэйд-монодромных разложения одному и тому же типу брэйд-разложения?

Одним из основных результатов данной статьи является следуюшая теорема.

ТЕОРема 1. Пусть $B_{1}, B_{2} \subset \mathbb{C P}^{2}$ - две каспидальные алгебраические кривие. Предположим, что парь $\left(\mathbb{C P}^{2}, B_{1}\right)$ u $\left(\mathbb{C P}^{2}, B_{2}\right)$ имеют один и тот жее тип брәйд-разложсения. Тогда пары $\left(\mathbb{C P}^{2}, B_{1}\right)$ и $\left(\mathbb{C P}^{2}, B_{2}\right)$ диффеоморфньи.

Хорошо известно, что существуют гомеоморфные, но не диффеоморфные, четырехмерные гладкие многообразия, и одной из наиболее важных проблем четырехмерной геометрии является проблема нахождения инвариантов, различающих гладкие структуры на одном и том же топологическом многообразии. Мы надеемся, что в алгебраическом случае тип брэйд-разложения дискриминантной кривой общего морфизма проективной поверхности $S$ на $\mathbb{C P}^{2}$ может быть использован в качестве такого инварианта гладкой структуры (индуцированной комплексной структурой) на $S$, рассматриваемой как четырехмерное действительное многообразие. Мы докажем следующую теорему. 
Teорема 2. Пусть $f_{1}: S_{1} \rightarrow \mathbb{C P}^{2}$ и $f_{2}: S_{2} \rightarrow \mathbb{C P}^{2}$ - два общих морфизма неособых проективных поверхностей и пусть $B_{1}, B_{2} \subset \mathbb{C P}^{2}$ - дискриминантные кривые этих морфизмов. Предположим, что гипотеза Кизини верна для $\left(\mathbb{C P}^{2}, B_{1}\right)$. Тогда если пары $\left(\mathbb{C P}^{2}, B_{1}\right)$ и $\left(\mathbb{C P}^{2}, B_{2}\right)$ имеют один и тот же тип брәйд-разложения, то $S_{1}$ и $S_{2}$ диффеоморфны.

СЛЕДСТВИЕ. Пусть $S_{1}$ и $S_{2}-$ две поверхности общего типа с обильнымм каноническим классом и пусть $B_{1}$ и $B_{2}-m$-канонические дискриминантные кривые соответственно общих морфизмов $f_{1}: S_{1} \rightarrow \mathbb{C P}^{2}$ и $f_{2}: S_{2} \rightarrow \mathbb{C P}^{2}$, заданных трехмерными линейными подсистемами из т-канонического класса на $S_{i}$, где $m \in \mathbb{N}$. Тогда если парьи $\left(\mathbb{C P}^{2}, B_{1}\right)$ и $\left(\mathbb{C P}^{2}, B_{2}\right)$ имеют один и тот же тип брэйд-разложения, то $S_{1}$ и $S_{2}$ диффеоморфны.

Опишем кратко содержание статьи по параграфам. В $\S \S 1-4$ мы напомним определения и некоторые факты, относяшиеся к "брэйд-монодромной технике", разработанной Б. Мойшезоном и вторым автором данной статьи. §5 посвящен описанию образующих централизатора кратного полуповорота в группе кос. Это описание является ключевым моментом для доказательства теоремы 1. В $\S 6$ мы напомним или докажем некоторые утверждения (возможно хорошо известные) о гладкой изотопии многообразий, которые будут использованы в доказательстве основных результатов. $\S 7$ посвящен доказательству теоремы 1 и в $\S 8$ мы докажем теорему 2 .

\section{§1. Брэйд-монодромия аффинной кривой}

В данной статье мы будем использовать следующие обозначения: $B$ - кривая в $\mathbb{C}^{2}$ или в $\mathbb{C P}^{2}, p=\operatorname{deg} B, \pi: \mathbb{C}^{2} \rightarrow \mathbb{C}$ - проекция на первую координату; $K(x)=$ $\{y \mid(x, y) \in B\} \quad\left(K(x)=\right.$ проекция на ось $y$ множества $\left.\pi^{-1}(x) \cap B\right) ; N=\{x \mid$ $\# K(x)<p\} ; M^{\prime}=\left\{x \in B|\pi|_{B}\right.$ не этально в точке $\left.x\right\}\left(\pi\left(M^{\prime}\right)=N\right)$.

Предположим, что \# $\left(\pi^{-1}(x) \cap M^{\prime}\right)=1$ для всех $x \in N$.

Пусть $E$ (соответственно $D$ ) - замкнутый диск на оси $x$ (соответственно на оси $y$ ) такой, что $M^{\prime} \subset E \times D, N \subset \operatorname{Int}(E)$ и что $\left.\pi\right|_{(E \times D) \cap B}$ является собственным морфизмом степени $p$.

Выберем $u \in \partial E$ и положим $K=K(u)=\left\{q_{1}, \ldots, q_{p}\right\}$.

В описанной выше ситуации можно ввести понятие "брэйд-монодромии".

ОПРЕДЕЛЕНИЕ (брәйд-монодромия кривой $B$ относительно $E \times D, \pi, u)$. Каждая петля $\gamma:[0,1] \rightarrow E \backslash N$ с началом и концом в точке $u$ может быть поднята до системы из $p$ путей в $(E \backslash N) \times D$ с началом в точках $q_{1}, \ldots, q_{p}$. Проектируя эти пути в $D$, мы получим $p$ путей в $D$, определяющих движение $p$ точек $\left\{q_{1}(t), \ldots, q_{p}(t)\right\}$ в $D$, начинающихся и заканчивающихся в $K$.

Это движение определяет косу в $B_{p}[D, K]$ (см. [6, гл.ІІІ]). Таким образом, мы получаем отображение $\varphi: \pi_{1}(E \backslash N, u) \rightarrow B_{p}[D, K]$. Это отображение, очевидно, является групповым гомоморфизмом и по определению называется брэйд-монодромией кривой $B$ относительно $E \times D, \pi, u$. Мы иногда будем обозначать $\varphi$ через $\varphi_{u}$.

ОПРЕДЕЛЕНИЕ (брәйд-монодромия кривой В относительно $\pi, u$ ). Рассматривая полученную вьше косу как элемент группы $B_{p}\left[\mathbb{C}_{u}, K\right]$, мы получаем гомоморфизмм $\varphi: \pi_{1}(E \backslash N, u) \rightarrow B_{p}\left[\mathbb{C}_{u}, K\right]$, который будем называть брэйд-монодромией кривой $B$ относительно $\pi, u$. Гомоморфизм $\varphi$ мы также иногда будем обозначать через $\varphi_{u}$. 
Чтобы привести пример вычисления брэйд-монодромии, мы напомним геометрическую модель группы кос и определение полуповорота.

ОПРЕДЕЛЕНИЕ (әрynna кос $B_{p}[D, K]$ ). Пусть $D$ - замкнутый диск в $\mathbb{R}^{2}, K \subset$ $D, K$-конечное множество. Пусть $\mathscr{B}$ - группа всех диффеоморфизмов $\beta$ диска $D$ таких, что $\beta(K)=K,\left.\beta\right|_{\partial D}=\operatorname{Id}_{\partial D}$. Мы скажем, что элемент $\beta_{1}$ эквивалентен $\beta_{2}$, если $\beta_{1}$ и $\beta_{2}$ индуцируют один и тот же автоморфизм группы $\pi_{1}(D \backslash K, u)$. Факторгруппа группы $\mathscr{B}$ по этому отношению эквивалентности назьвается группой кос $B_{p}[D, K](p=\# K)$. Элементы группы $B_{p}[D, K]$ называются косами. Обозначим через $\bar{\beta}$ косу, представленную диффеоморфизмом $\beta$.

ОПРЕДЕЛЕНИЕ ( полуповорот $H(\sigma)$, заданный путем $\sigma$ ). Пусть $D, K$ - как и выше, $a, b \in K, K_{a, b}=K \backslash\{a, b\}$, и пусть $\sigma$ - простой (т. е. без самопересечений) путь в $D \backslash \partial D$, соединяюший $a$ с $b$, такой, что $\sigma \cap K=\{a, b\}$. Выберем некоторую достаточно маленькую окрестность $U$ пути $\sigma, K_{a, b} \cap U=\varnothing$, а также сохраняющий ориентацию диффеоморфизм $\psi: \mathbb{R}^{2} \longrightarrow \mathbb{C}^{1}\left(\mathbb{C}^{1}\right.$ взято с обычной “комплексной" ориентацией) так, что $\psi(\sigma)=[-1,1]=\left\{z \in \mathbb{C}^{1} \mid \Re z \in[-1,1], \Im z=0\right\}$ и $\psi(U)=\left\{z \in \mathbb{C}^{1}|| z \mid<2\right\}$. Пусть $\alpha(r), r \geqslant 0,-$ гладкая монотонная вешественная функция такая, что $\alpha(r)=1$ для $r \in\left[0, \frac{3}{2}\right]$ и $\alpha(r)=0$ для $r \geqslant 2$. Рассмотрим диффеоморфизм $h: \mathbb{C}^{1} \rightarrow \mathbb{C}^{1}$, действующий на $z \in \mathbb{C}^{1}, z=r e^{i \varphi}$, по правилу $h(z)=r e^{i(\varphi+\alpha(r) \pi)}$. Очевидно, что ограничение $h$ на $\left\{z \in \mathbb{C}^{1}|| z \mid \leqslant \frac{3}{2}\right\}$ совпадает с поворотом на угол $\pi$, а ограничение на $\left\{z \in \mathbb{C}^{1}|| z \mid \geqslant 2\right\}$ является тождественным диффеоморфизмом. Диффеоморфизм $H(\sigma)=\psi^{-1} \circ h \circ \psi$ будем называть полуповоротом.

Полуповорот $H(\sigma)$ определяет геометрическую косу $\bar{\sigma}$ (т.е. $p$ непересекаюшихся путей в $D \times[0,1]$ с началами в $K \times\{0\}$ и с концами в $K \times\{1\}, K=$ $\left.\left\{q_{1}, \ldots, q_{p}\right\}\right)$. Эта коса задается следующими формулами

$$
\begin{aligned}
& \left(\delta_{j}(t), t\right)=\left(q_{j}, t\right), \quad \text { если } q_{j} \neq a, b ; \\
& \left(\delta_{j}(t), t\right)=\left(\psi^{-1}\left(e^{\pi i t}\right), t\right), \quad \text { если } q_{j}=a ; \\
& \left(\delta_{j}(t), t\right)=\left(\psi^{-1}\left(-e^{\pi i t}\right), t\right), \quad \text { если } q_{j}=b \text {. }
\end{aligned}
$$

Следуюшее предложение является основным примером брэйд-монодромии, связанным с простой двойной особой точкой алгебраической кривой.

ПРЕДЛОЖЕНИЕ-ПРИМЕР 1.1. Пусть $E=\{x \in \mathbb{C}|| x \mid \leqslant 1\}, \quad D=\{y \in$ $\mathbb{C} \mid y \leqslant R\}, \quad R \gg 1, \quad B$ - кривая, заданная уравнением $y^{2}=x^{\nu}, \quad u=1$. Ясно, что в этом случае $p=2, \quad N=\{0\}, \quad K=\{-1,+1\}$ u $\pi_{1}(E \backslash N, 1)$ порождается петлей $\Gamma=\partial E$ ( с положительной ориентацией). Обозначим $\varphi: \pi_{1}(E \backslash N, 1) \rightarrow B_{2}[D, K]$ брәйд-монодромию кривой $B$ относительно $E \times D$, $\pi$, и. Тогда $\varphi(\Gamma)=H^{\nu}$, где $H$ - положительный полуповорот, заданный путем $[-1,1]$ ( "положительная образующая" группы $B_{2}[D, K]$ ).

ДоказАтельство. Мы можем записать $\Gamma=\left\{e^{2 \pi i t}, t \in[0,1]\right\}$. Поднимая $\Gamma$ на $B$, мы получим два пути:

$$
\delta_{1}(t)=\left(e^{2 \pi i t}, e^{2 \pi i \nu t / 2}\right), \quad \delta_{2}(t)=\left(e^{2 \pi i t},-e^{2 \pi i \nu t / 2}\right) .
$$

Проектируя $\delta_{1}(t), \delta_{2}(t)$ на $D$, мы получим два пути:

$$
a_{1}(t)=e^{\pi i t \cdot \nu}, \quad a_{2}(t)=-e^{\pi i t \cdot \nu}, \quad 0 \leqslant t \leqslant 1 .
$$


Эти пути задают движение точек $\{1,-1\}$ в $D$. Это движение есть $\nu$-я степень движения $\mathscr{M}$ :

$$
b_{1}(t)=e^{\pi i t}, \quad b_{2}(t)=-e^{\pi i t}, 0 \leqslant t \leqslant 1 .
$$

Коса в $B_{2}[D,\{1,-1\}]$, индуцированная движением $\mathscr{M}$, совпадает с полуповоротом $H$, соответствуюшим пути $[-1,1] \subset D$. Таким образом, $\varphi(\Gamma)=H^{\nu}$.

Напомним определение и некоторые основные свойства геометрического свободного базиса фундаментальной группы проколотого диска.

ОПРЕДЕЛЕНИЕ $(\kappa y c m)$. Пусть $E, N=\left\{u_{1}, \ldots, u_{n}\right\}, u$ определены так же, как и выше. Рассмотрим в $E$ упорядоченное множество простых путей $\left(T_{1}, \ldots, T_{n}\right)$, соединяюших точки $u_{i}$ с точкой $u$ так, что

1) $T_{i} \cap T_{j}=u$, если $i \neq j$;

$2)$ для некоторой окружности $c(u)$ маленького радиуса с центром в $u$ каждое пересечение $T_{i} \cap c(u)$ состоит из одной точки, скажем $w_{i}$, и порядок нумерации в $\left(w_{1}, \ldots, w_{n}\right)$ совпадает с порядком, индуцированным положительной ("против часовой стрелки") ориентацией на $c(u)$.

Пусть $c_{j}-$ граница замкнутого диска $E_{j}$ с центром в точке $u_{j}$ достаточно малого радиуса. Обозначим $T_{j}^{\prime}=T_{j} \backslash\left(T_{j} \cap E_{j}\right)$ и $l\left(T_{j}\right)=T_{j}^{\prime} \cdot c_{j} \cdot T_{j}^{\prime-1}$ замкнутую петлю (а также соответствуюший элемент в $\pi_{1}(E \backslash N, u)$ ), в которой окружность $c_{j}$ обходится в направлении против часовой стрелки.

Мы скажем, что два таких множества путей $\left(T_{1}, \ldots, T_{n}\right)$ и $\left(\widetilde{T}_{1}, \ldots, \widetilde{T}_{n}\right)$ являются эквивалентньми, если для всех $i=1, \ldots, n$

$$
\ell\left(T_{i}\right)=\ell\left(\widetilde{T}_{i}\right) \quad \text { в } \quad \pi_{1}(E \backslash N, u) .
$$

Класс эквивалентности таких множеств называется кустом в $(E \backslash N, u)$. Куст, представленный множеством $\left(T_{1}, \ldots, T_{n}\right)$, будет обозначаться $\left\langle T_{1}, \ldots, T_{n}\right\rangle$.

ОПРЕДЕЛЕНИЕ (геометрический базис, $g$-базис). Пусть $E, N, u$ определены так же, как и ранее. Назовем $g$-базисом группы $\pi_{1}(E \backslash N, u)$ упорядоченный свободный базис $\left(\ell\left(T_{1}\right), \ldots, \ell\left(T_{n}\right)\right)$ группы $\pi_{1}(E \backslash N, u)$, где $\left\langle T_{1}, \ldots, T_{n}\right\rangle$ является кустом в $E \backslash N$ (см. рис. 1.1).

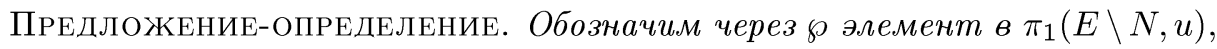
представленной петлей $\partial E$ ( п положительной ориентацией). Существует единственный әлемент в $B_{n}[E, N]$, обозначаемый $\Delta_{n}^{2}$ или $\Delta_{n}^{2}[E, N]$, такой, что для любого g-базиса $\Gamma_{1}, \ldots, \Gamma_{n}$ группы $\pi_{1}(E \backslash N, u)$

$$
\left(\Gamma_{i}\right) \Delta_{n}^{2}=\wp \Gamma_{i \wp} \wp^{-1} .
$$

ДокаЗАТЕЛЬСТво. См. [6, V.2.1].

ЗАмечАнИЕ. Ясно, что $\Delta_{n}^{2}$ действует на $E$ как полньй поворот вокруг всех точек из $N$.

ПРЕДЛОЖЕНИЕ 1.1. Элемент $\Delta_{n}^{2}$ принадлежит центру группы $B_{n}[E, N]$.

ДОКАЗАТЕЛЬСТво. См. [6, V.4.1]. 


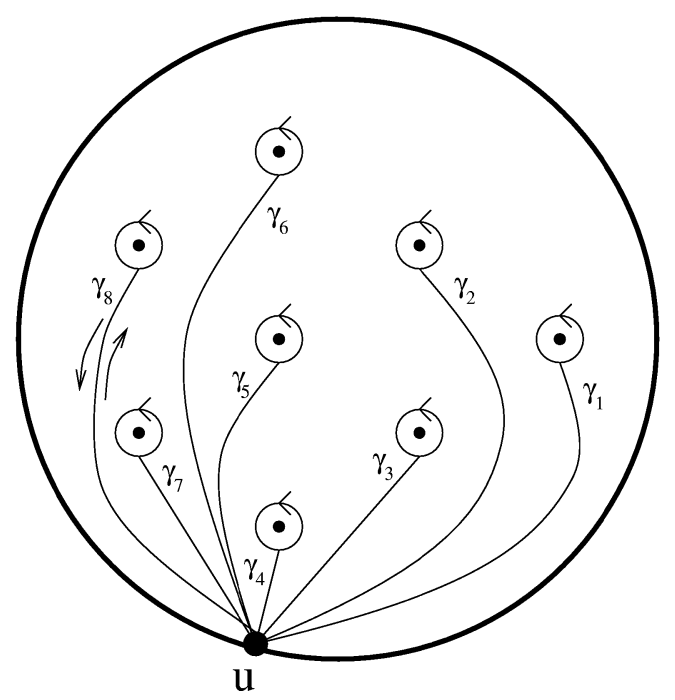

Рис. 1.1

ПРЕДЛОЖЕНИЕ-ПРИМЕР 1.2. Пусть В - оббединение $p$ прямых, пересекающихся в одной точке $s_{0}, s_{0}=\left(x\left(s_{0}\right), y\left(s_{0}\right)\right)$. Пусть $D, E, u, K=K(u)-$ как и выше, и пусть $\varphi-$ брәйд-монодромия кривой $B$ относительно $E \times D$, $\pi, u$. Ясно, что $N$ состоит из одной точки $x\left(s_{0}\right)$ u $\pi_{1}(E \backslash N, u)$ порождается петлей $\Gamma=\partial E$. Тогда $\varphi(\Gamma)=\Delta_{p}^{2}=\Delta_{p}^{2}[D, K(u)]$.

ДокАЗАТЕЛЬСтво. Непрерьвно меняя $s_{0}$ и $p$ прямых, проходяших через $s_{0}$ (а также в силу единственности $\Delta_{p}^{2}$ ), мы можем свести доказательство к случаю $B=$ $\cup L_{k}, L_{k}=\left\{(y, x) \in \mathbb{C}^{2} \mid y=j_{k} x, j_{k}=e^{2 \pi i k / p}, k=0, \ldots, p-1\right\}$. Тогда $N=\{0\}$. Мы можем взять $E=\left\{x \in \mathbb{C}^{1}|| x \mid \leqslant 1\right\}, u=1, \Gamma=\partial E=\left\{x=e^{2 \pi i t}, t \in[0,1]\right\}$. Поднимая $\partial E$ на $B$ и затем проектируя его на $D$, мы получим $p$ петель:

$$
a_{k}(t)=e^{2 \pi i(t+k / p)}, \quad k=0, \ldots, p-1, \quad t \in[0,1] .
$$

Движение точек $a_{k}(0)$ вдоль петель $a_{k}(t)$ является полным поворотом, которьй определяет косу $\Delta_{p}^{2}\left[D,\left\{a_{k}(0)\right\}\right]=\Delta_{p}^{2}[D, K(1)]$. (Чтобы проверить последнее утверждение, надо рассмотреть соответствуюшее действие на группе $\pi_{1}(D \backslash K, v)$, где $v \in \partial D$.)

ОПРЕДЕЛЕНИЕ (остов аруппы кос $B_{p}[D, K]$ ). Пусть $D, K=\left\{q_{1}, \ldots, q_{p}\right\}-$ как и выше. Выберем систему простых гладких путей $\left(\sigma_{1}, \ldots, \sigma_{p-1}\right)$ в $D \backslash \partial D$, соединяюших точки $q_{i}$ с $q_{i+1}$ так, что $L=\cup \sigma_{j}$ является простым гладким путем. Упорядоченная система полуповоротов $\left(H_{1}, \ldots, H_{p-1}\right)$, заданных путями $\left\{\sigma_{i}\right\}_{i=1}^{p-1}$, называется остовом группы кос $B_{p}[D, K]$. Иногда такую систему путей $\left\{\sigma_{i}\right\}_{i=1}^{p-1}$ мы также будем называть остовом группы $B_{p}[D, K]$.

TeOpema 1.1. Пусть $\left(H_{1}, \ldots, H_{p-1}\right)$ - ocmos грynnь $B_{p}[D, K] . \quad$ Тогда $B_{p}[D, K]$ порождается әлементами $H_{1}, \ldots, H_{p-1}$.

ДокАЗАТЕЛЬСТво можно найти, например, в [6]. 
ОПРЕДЕЛЕНИЕ (обобщенный полуповорот $\Delta_{i, j}$, заданный путем $L_{i, j}=$ $\left.\bigcup_{r=i}^{j-1} \sigma_{r}, i<j\right)$. В обозначениях из предыдущего определения пусть $\left\{\sigma_{r}\right\}_{r=1}^{p-1}-$ остов группы $B_{p}[D, K]$, и пусть $i<j$. Выберем некоторую достаточно маленькую окрестность $U$ пути $L_{i, j}$, а также сохраняюший ориентацию диффеоморфизм $\psi: \mathbb{R}^{2} \longrightarrow \mathbb{C}^{1}$ так, что

1) $\psi\left(L_{i, j}\right)=[-1,1]=\left\{z \in \mathbb{C}^{1} \mid \operatorname{Re} z \in[-1,1], \operatorname{Im} z=0\right\}$;

2) $\psi(U)=\left\{z \in \mathbb{C}^{1}|| z \mid<2\right\}$

3) множество $\left\{\psi\left(q_{r}\right)\right\}_{r=i}^{j}$ инвариантно относительно инволюции $\operatorname{Re} z \mapsto-\operatorname{Re} z$.

Пусть $\alpha(r), r \geqslant 0,-$ гладкая монотонная вешественная функция такая, что $\alpha(r)=1$ для $r \in\left[0, \frac{3}{2}\right]$ и $\alpha(r)=0$ для $r \geqslant 2$. Рассмотрим диффеоморфизм $h: \mathbb{C}^{1} \rightarrow \mathbb{C}^{1}$, действующий на $z \in \mathbb{C}^{1}, z=r e^{i \varphi}$, по правилу $h(z)=r e^{i(\varphi+\alpha(r) \pi)}$. Диффеоморфизм $\Delta_{i, j}=\psi^{-1} \circ h \circ \psi$ будем называется полуповоротом, заданным путем $L_{i, j}=\bigcup_{r=i}^{j-1} \sigma_{r}$.

ЗАмечаниЕ. Полный поворот $\Delta_{i, j}^{2}=\left(H_{i} \cdot \ldots \cdot H_{j-1}\right)^{j-i+1}$. В частности, $\Delta_{i, i+1}=H_{i}$ и $\Delta_{1, p}^{2}=\Delta_{p}^{2}$, где полуповорот $H_{i}$ и полньй поворот $\Delta_{p}^{2}=\Delta_{p}^{2}[D, K]$ были определены вьше.

\section{§ 2. Положительность брэйд-монодромии}

В этом параграфе мы покажем, что брэйд-монодромия принимает значения в полугруппе $B_{p}^{+}$, порожденной положительными (против часовой стрелки) полуповоротами. Мы сохраним те же самые обозначения, что и в $§ 1$, и введем несколько дополнительных.

ОПРЕДЕЛЕНИЕ (диффеоморфизм Лефшеца $\psi_{T}$, индуиированный путем $T$ ). Пусть $T$-путь в $E \backslash N$, соединяющий точку $x_{0}$ с $x_{1}, T:[0,1] \rightarrow E \backslash N$. Сушествует непрерывное семейство диффеоморфизмов $\psi_{(t)}: D \rightarrow D, t \in[0,1]$, такое, что $\psi_{(0)}=\operatorname{Id}, \psi_{(t)}\left(K\left(x_{0}\right)\right)=K(T(t))$ для всех $t \in[0,1]$ и $\psi_{(t)}(y)=y$ для всех $y \in \partial D$. Чтобы подчеркнуть зависимость от $t$, мы будем писать $\psi_{(t)}:\left(D, K\left(x_{0}\right)\right) \rightarrow$ $(D, K(T(t)))$,

$$
\psi_{T}=\psi_{(1)}:\left(D, K\left(x_{0}\right)\right) \underset{\sim}{\sim}\left(D, K\left(x_{1}\right)\right) .
$$

Так как $\psi_{(t)}\left(K\left(x_{0}\right)\right)=K(T(t))$ для всех $t \in[0,1]$, мы имеем семейство канонических изоморфизмов $\psi_{(t)}^{\nu}: B_{p}\left[D, K\left(x_{0}\right)\right] \underset{\sim}{\longrightarrow} B_{p}[D, K(T(t))] \forall t \in[0,1]$.

ОПРЕДЕЛЕНИЕ ( изоморфизм Лефиеца $L_{T}$, индуцированный путем $T$ ).

$$
\begin{aligned}
L_{T} & =\psi_{T}^{\nu}=\psi_{(1)}^{\nu}: B_{p}\left[D, K\left(x_{0}\right)\right] \\
(\bar{\beta}) L_{T} & =\overline{\psi_{T}^{-1} \circ \beta \circ \psi_{T}} .
\end{aligned}
$$

Легко проверить, что $L_{T}$ зависит только от гомотопического класса пути $T$.

Изоморфизмы $L_{T}$ и $\psi_{T}$ зависят не только от $T$, но также и от кривой $B$. В случае, когда рассматривается несколько кривых, мы будем использовать обозначение $\psi_{T, B}$ и $L_{T, B}$, чтобы подчеркнуть их зависимость от $B$.

ЗАмЕчАниЕ. Существует другое, эквивалентное определение брэйд-монодромии $\varphi_{u}: \pi_{1}(E \backslash N, u) \rightarrow B_{p}[D, K]$. Возьмем любой элемент $\delta \in \pi_{1}(E \backslash N, u)$. Пусть $\underline{\delta}$ - петля, представляющая $\delta$. Тогда, по определению, $\varphi(\delta)$ есть коса, представленная дифффеоморфизмом $\psi_{\underline{\delta}}$. 
ДокаЗАТЕЛЬСТво. Оба $\psi_{\underline{\delta}}$ и $\varphi(\delta)$ индуцированы “движением" вдоль $\delta$.

ЛЕмма 2.1. Имеют место следующие утверждения:

1) $\psi_{T_{1} T_{2}}=\psi_{T_{1}} \circ \psi_{T_{2}}$

2) $H(\sigma) L_{t}=H\left((\sigma) \psi_{T}\right)$;

3) если $T$ - замкнутая петля, то $\psi_{T}$ определяет косу $\bar{\psi}_{T}$ в $B_{p} u(b) L_{T}=$

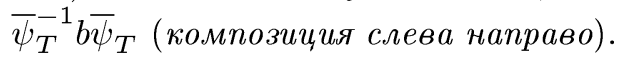

ДокАЗАТЕЛЬство. Утверждения 1) и 2) очевидны.

3) Предположим $b=\bar{\beta}$. По определению изоморфизма Лефшеца $L_{T},(\bar{\beta}) L_{T}=$ $\overline{\psi_{T}^{-1} \circ \beta \circ \psi_{T}}$. Так как $\psi_{T}$ и $\beta$ определяют косу, то $(b) L_{T}=\bar{\psi}_{T}^{-1} \circ \bar{\beta} \circ \bar{\psi}_{T}=\bar{\psi}_{T}^{-1} b \bar{\psi}_{T}$.

Пусть $M^{\prime}=\left\{s_{j}\right\}_{j=1}^{n}$ - особые точки проекции $\left.\pi\right|_{B}, \pi\left(M^{\prime}\right)=N$. Для каждого $j=1, \ldots, n$ пусть $D_{j}^{\prime}$ - маленький диск на оси $y$ с центром в $y\left(s_{j}\right)$ такой, что $D_{j} \subseteq D$ и такой, что $\left(x\left(s_{j}\right) \times D_{j}^{\prime}\right) \cap B=s_{j}$. Пусть $E_{j}^{\prime}$ - достаточно маленькая окрестность точки $x_{j}=x\left(s_{j}\right)$ на оси $x$ такая, что $E_{j}^{\prime} \cap N=x_{j}$ и для любого $x \in E_{j}^{\prime} \backslash\left\{x_{j}\right\}$ число $\#\left\{\left(x \times \operatorname{Int}\left(D_{j}^{\prime}\right)\right) \cap B\right\}$ не зависит от $x$. Мы будем называть это число локальной степенью проекции $\pi$ в точке $s_{j}$ и обозначать $\operatorname{deg}_{s_{j}} \pi$. Пусть $m_{j}=\operatorname{deg}_{s_{j}} \pi$. Выберем точку $x_{j}^{\prime} \in \partial E_{j}^{\prime}$. Положим $K^{\prime}\left(x_{j}^{\prime}\right)=K\left(x_{j}^{\prime}\right) \cap D_{j}^{\prime}$.

ОПРЕДЕЛЕНИЕ (вложение Лефшеца $\psi_{T, s_{j}}$, индущированное $s_{j} u T$ ). Пусть $T:[0,1] \rightarrow \mathbb{C}$-путь в $E \backslash\left(N \cup\left(\operatorname{Int} E_{j}^{\prime}\right)\right)$, соединяюший $x_{j}^{\prime}$ с точкой $u^{\prime} \in E \backslash N$. Дифофеоморфизм $\psi_{T, s_{j}}=\left.\psi_{T}\right|_{D_{j}^{\prime}}:\left(D_{j}^{\prime}, K^{\prime}\left(x_{j}^{\prime}\right)\right) \rightarrow\left(D, K\left(u^{\prime}\right)\right)$ называется вложением Лефшшеца, индуцированным путем $T$ и точкой $s_{j}$.

ЗАмечаниЕ. Пусть $m_{j}=\operatorname{deg}_{s_{j}} \pi$. Возьмем $m_{j}$ подъемов пути $T$ на $B$, начинающихся в различных точках из $K^{\prime}\left(x_{j}^{\prime}\right)$. Эти подъемы являются вещественными кривыми в $T \times D$. Мы можем рассматривать $\psi_{T}$ как “перетаскивание" $K^{\prime}\left(x_{j}^{\prime}\right)$ внутри $T \times D$ вдоль этих вешественных кривых.

ОПРЕДЕЛЕНИЕ (мономорфизм Лефиеца $L_{T, s_{j}}$, индуиированный путем $T$ ). Пусть $s_{0} \in N$, и пусть $D_{0}^{\prime}, x_{0}, E_{0}, m_{0}, u^{1}$ - те же, что и в определении вложения Лефшеца. Рассмотрим вложение Лефшеца $\psi_{T, s_{j}}:\left(D_{j}^{\prime} \times K\left(x_{j}^{\prime}\right)\right) \rightarrow\left(D, K\left(u^{\prime}\right)\right)$, индуцированное $s_{j}$ и $T$. Имеем

$$
\psi_{T, s_{j}}\left(K^{\prime}\left(x_{j}^{\prime}\right)\right) \subset K\left(u^{\prime}\right) \text { и }\left(K\left(u^{\prime}\right) \backslash \psi_{T, s_{j}}\left(K\left(x_{j}^{\prime}\right)\right) \cap \psi_{T, s_{j}}\left(\operatorname{Int} D_{j}^{\prime}\right)=\phi .\right.
$$

Мономорфизм Лефшеца, индуцированный путем $T$, есть канонический, корректно определенный гомоморфиим

$$
L_{T}=L_{T, s_{j}}=\psi_{T}^{\nu}: B_{m_{j}}\left[D_{j}^{\prime}, K^{\prime}\left(x_{j}^{\prime}\right)\right] \hookrightarrow B_{p}\left[D, K\left(u^{\prime}\right)\right]
$$

индуцированный $\psi_{T, s_{j}}$ и рассмотренными выше включениями.

Чтобы вычислить брэйд-монодромию, мы должны знать $\left\{\varphi\left(\delta_{j}\right)\right\}_{j=1}^{n}$ для $g$-базиса $\left\{\delta_{j}=\ell\left(\gamma_{j}\right)\right\}_{j=1}^{n}$, определяемого кустом $\left\{\gamma_{j}\right\}_{j=1}^{n}$ в $(E \backslash N, u)$. Мы можем представить $\delta_{j}$ в виде $\tilde{\gamma}_{j}^{-1} \circ \partial E_{j}^{\prime} \circ \tilde{\gamma}_{j}\left(E_{j}^{\prime}\right.$ были определены ранее). Таким образом, чтобы знать $\varphi\left(\delta_{j}\right)$, достаточно знать мономорфизм Лефшеца $L_{\tilde{\gamma}_{j}}: B_{m_{j}}\left[D_{j}^{\prime}, K^{\prime}\left(x_{j}^{\prime}\right)\right] \rightarrow$ $B_{p}[D, K]$ и локальную брэйд-монодромию $\varphi_{s_{j}}$ кривой $B \cap\left(E_{j}^{\prime} \times D_{j}^{\prime}\right)$ относительно $E_{j}^{\prime} \times D_{j}^{\prime}, \pi, x_{j}^{\prime}$. 
ОПРЕДЕЛЕНИЕ (локальная брәйд-монодромия $\varphi_{s_{j}}$ кривой $B$ в точке $s_{j}$ ). Гомоморфизм $\varphi_{s_{j}}: \pi_{1}\left(E_{j}^{\prime} \backslash\left\{x_{j}\right\}, x_{j}^{\prime}\right) \rightarrow B_{m_{j}}\left[D_{j}^{\prime}, K^{\prime}\left(x_{j}^{\prime}\right)\right]$ называется локальной брэйдмонодромией кривой $B \cap\left[E_{j}^{\prime} \times D_{j}^{\prime}\right]$ относительно $E_{j}^{\prime} \times D_{j}^{\prime}, \pi, x_{j}^{\prime}\left(K^{\prime}\left(x_{j}^{\prime}\right)=\right.$ $\left.K\left(x_{j}^{\prime}\right) \cap D_{j}^{\prime}\right)$

Ясно, что $\varphi_{s_{j}}$ определяется элементом $\varphi_{s_{j}}\left(\partial E_{j}^{\prime}\right)$. Имеет место следуюшая лемма.

ЛЕмма 2.2. Пусть $T$ - произвольный путь в $E \backslash\left(N \cup \operatorname{Int}\left(E_{j}^{\prime}\right)\right)$, соединяющий $x_{j}^{\prime}$ c u, и пусть $\delta=T^{-1} \circ \partial E_{j}^{\prime} \circ T=\ell(T)$. Тогда $\varphi(\delta)=\left(\varphi_{s_{j}}\left(\partial E_{j}^{\prime}\right)\right) L_{T} . \quad B$ частности, $\varphi\left(\delta_{j}\right)=\left(\varphi_{s_{j}}\left(\partial E_{j}^{\prime}\right)\right) L_{\tilde{\gamma}_{j}}$.

ЗАмЕчАниЕ. Лемма фактически показывает, что брэйд-монодромия $\varphi$ полностью определена, если мы знаем локальные брэйд-монодромии и мономорфизмы Лефшшеца для некоторого куста в $E \backslash N$.

ЛЕмма 2.3. Пусть $s_{j}$-особая точка кривой $B$ с локальным уравнением вида $y^{2}=x^{\nu}$, m.е. $m_{j}=\operatorname{deg}_{s_{j}} \pi=2$. Тогда $\varphi\left(\delta_{j}\right)=\left(H_{j}\right)^{\nu}$, где $H_{j}$ - положительный полуповорот в $B_{p}[D, K(u)]$, заданный некоторым путем $\sigma, u, \boldsymbol{\theta}$ частности, является положительной косой.

ДоКАЗАТЕЛЬСТВО следует из леммы 2.2 и предложения-примера 1.1.

ПрЕДЛОЖенИЕ 2.1. Пусть $\varphi: \pi_{1}(E \backslash N, u) \rightarrow B_{p}[D, K]-$ брэйд-монодромия кривой $B$ и $\left\{\delta_{j}\right\}-g$-базис группьи $\pi_{1}(E \backslash N, u)$. Тогда $\varphi\left(\delta_{j}\right) \in B_{p}^{+}=B_{p}^{+}[D, K]$ для всех $j$.

ДОКАЗАТЕЛЬСТВо. Для данной кривой $B$ мы можем найти достаточно близкую к $B$ неособую кривую $B^{(1)}$ той же степени. Пусть $K^{(1)}=\left\{y \mid(u, y) \in B^{(1)}\right\}$ и $M^{(1)}, N^{(1)}$ - те же, что и в $\S 1$. Ввиду близости кривой $B$ с $B^{(1)}$ мы можем отождествить $B_{p}[D, K]$ с $B_{p}\left[D, K^{(1)}\right]$. Каждая точка $s_{j} \in M$ рассьпается на конечное число особых точек $\left\{s_{j i}\right\} \subseteq M^{(1)}$, каждая из которых имеет локальное уравнение $x=y^{2}$. Каждая точка $x_{j} \in N$ рассыпется на точки $\left\{x_{j i}\right\}_{i}=\left\{\pi\left(s_{j i}\right)\right\}_{i} \subseteq N^{(1)}$. Ясно, что $N^{(1)}=\left\{x_{j i}\right\}_{j, i}$. Пусть $\varphi^{(1)}$ - брэйд-монодромия кривой $B^{(1)}$ относительно $E \times D, \pi, u, N^{(1)}$. Мы можем найти $g$-базис $\left\{\delta_{j i}\right\}$ группы $\pi_{1}\left(E \backslash N^{(1)}, u\right)$ такой, что $\delta_{j}=\prod_{i} \delta_{j i}$ для каждого $j$. Естественное отождествление групп $B_{p}[D, K]$ и $B_{p}\left[D, K^{(1)}\right]$ приводит нас к равенствам $\varphi\left(\delta_{j}\right)=\prod_{i} \varphi^{(1)}\left(\delta_{j i}\right)$ (мы используем тот факт, что $B^{(1)}$ очень близка к $B$ ). По лемме 2.3 каждый $\varphi^{(1)}\left(\delta_{j i}\right)$ является положительным полуповоротом. Таким образом, $\varphi\left(\delta_{j}\right)=\prod_{i} \varphi^{(1)}\left(\delta_{j i}\right) \in B_{p}^{+}$для каждого $j$.

\section{§3. Брэйд-монодромия проективной кривой}

ОПРЕДЕЛЕНИЕ (брәйд-монодромия проективной кривой). Пусть $B$-алгебраическая кривая степени $p$ в $\mathbb{C P}^{2}$. Выберем бесконечно удаленную прямую $L$, находящуюся в общем положении с $B(\#(L \cap B)=p)$, а также выберем аффинные координаты $(x, y)$ в $\mathbb{C}^{2}=\mathbb{C P}^{2} \backslash L$ так, что проекция $\pi(x, y)=x$ на ось $x$ кривой $B \cap \mathbb{C}^{2}$ есть общая проекция (в частности, центр этой проекции в $\mathbb{C P}^{2}$ должен лежать вне $B$ ). Пусть $N=\left\{x \in \mathbb{C} \mid \pi^{-1}(x) \cap B \lesseqgtr p\right\}, E$ - замкнутьй диск на оси $x$ такой, что $N \subset \operatorname{Int}(E)$ и $D$ - замкнутый диск на оси $y$ такой, что $\pi^{-1}(E) \cap B \subset E \times D$. 
Выберем $u \in \partial E$. По определению брэйд-монодромия кривой $B$ относительно $L$, $u$ есть брэйд-монодромия кривой $B \cap(E \times D)$ относительно $E \times D, \pi, u$, т. е. гомоморфизм

$$
\varphi: \pi_{1}(E \backslash N, u) \rightarrow B_{p}[D, K]
$$

определенный в $\S 1$.

ПрЕДЛОЖЕНИЕ 3.1. Пусть $B$ - алгебраическая кривая степени р в $\mathbb{C P}^{2}$.

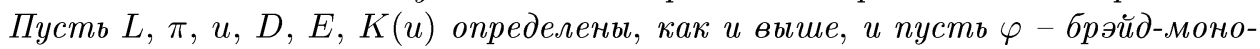
дромия кривой $B$ относительно $L, \pi, u$. Пусть $\delta_{1}, \ldots, \delta_{q}-g$-базис группь $\pi_{1}(E \backslash N, u)$. Тогда

$$
\prod_{i=1}^{q} \varphi\left(\delta_{i}\right)=\Delta_{p}^{2}=\Delta_{p}^{2}[u \times D, K(u) \cap B] .
$$

ДокАЗАТЕЛЬСТво. Так как $\prod_{i=1}^{q} \delta_{i}=\partial E$ (с положительной ориентацией), то мы должны доказать, что $\varphi(\partial E)=\Delta_{p}^{2}$. Мы можем считать, что диск $E$ является достаточно большим, так что $\partial E$ достаточно близка к $\infty$. Непрерьвно меняя коэффициенты уравнения кривой $B$ так, что все кривые из этого непрерывного семейства трансверсально пересекают прямую $L_{\infty}$, мы можем свести доказательство к случаю $p$ прямых, пересекаюшихся в одной точке, и применить предложение-пример 1.2.

ЛЕмма 3.1. Элемент $\Delta_{p}^{2} \in B_{p}^{+}$.

ДОКАЗАТЕЛЬСТВО следует из предложения 2.1.

ОПРЕДЕЛЕНИЕ (брәйд-монодромное разложение). Брэйд-монодромным разложением на множители, связанным с плоской проективной кривой, называется разложение $\Delta_{p}^{2}$ в произведение вида $\Delta_{p}^{2}=\prod_{i} \varphi\left(\delta_{i}\right)$, где $\varphi$ - брэйд-монодромия проективной кривой и $\left\{\delta_{j}\right\}$ - некоторый $g$-базис группы $\pi_{1}\left(E \backslash N_{1}, u\right)$.

ЗАмечАния. 1) Выбор $g$-базиса в $\pi_{1}(E \backslash N, u)$ и соответствуюшее этому базису разложение элемента $\Delta_{p}^{2}$ в виде произведения определяют брэйд-монодромию кривой $B$. Для приложений обычно достаточно знать только это брэйд-монодромное разложение без ссылки на фиксацию $g$-базиса.

2) Разложение элемента $\Delta_{p}^{2}$ в виде произведения не является разложением на неприводимые элементы из полугруппы $B_{p}^{+}$за исключением случая, когда $B$ является неособой кривой. В случае если $B-$ неособая кривая, то каждый $\varphi\left(\delta_{i}\right)$ является положительным полуповоротом и эти элементы неприводимы в $B_{p}^{+}$.

ПрЕДЛОЖЕНИЕ 3.2. Пусть $B$ - (обобщенная) каспидальная кривая в $\mathbb{C P}^{2}$ (т. е. все особые точки кривой $B$ являются особьми точками типа $y^{2}=x^{\nu}$, $\nu \in \mathbb{N}$ ). Тогда любое брәйд-монодромное разложение можст быть записано в виде $\Delta_{p}^{2}=\prod_{i}\left(Q_{i}^{-1} H_{1}^{\nu_{i}} Q_{i}\right)$, где $H_{1}$ - положительный полуповорот и каждое $\nu_{i} \in \mathbb{N}$

ДокАЗАТЕЛЬСТво. Напомним, что мы рассматриваем общую относительно проективной кривой $B$ проекцию $\mathbb{C}^{2} \stackrel{\pi}{\rightarrow} \mathbb{C}$. Каждая особенность отображения $\left.\pi\right|_{B}$ имеет тип $y^{2}=x_{i}^{\nu}, \nu_{i} \in \mathbb{N}$. Из леммы 2.3 следует, что $\varphi\left(\delta_{i}\right)=H_{i}^{\nu_{i}}$, где $H_{i}-$ некоторый полуповорот. Каждые два полуповорота в $B_{p}$ сопряжены друг другу, поэтому для всех $i$ существует $Q_{i}$ такое, что $H_{i}=Q_{i}^{-1} H_{1} Q_{i}$. Таким образом, $\Delta_{p}^{2}=\Pi \varphi\left(\delta_{i}\right)=\Pi Q_{i}^{-1} H_{1}^{\nu_{i}} Q_{i}$.

ЗАмечАНИЕ. В качестве $H_{1}$ мы можем взять любой полуповорот. 


\section{§4. Тип разложения брэйд-монодромии}

Согласно предложениям 3.1 и 2.1 мы знаем, что брэйд-монодромное разложение $\Delta_{p}^{2}[D, K]=\prod_{i} \varphi\left(\delta_{i}\right)$ является разложением на множители в $B_{p}^{+}[D, K]$ элемента $\Delta_{p}^{2}[D, K]$, которое связано с некоторым $g$-базисом групшы $\pi_{1}(E \backslash N, u)$. Определим отношение эквивалентности на множестве $B_{p}^{+}$-разложений на множители элемента $\Delta_{p}^{2}$. Для этого мы определим замены Гурвица на $G \times \cdots \times G$ (где $G-$ группа) и на множестве разложений на множители.

ОПРЕДЕЛЕНИЕ (замены Гурвица $R_{k} u R_{k}^{-1}$ на $G^{m}$ ). Пусть $\vec{t}=\left(t_{1}, \ldots, t_{m}\right) \in$ $G^{m}$. Мы скажем, что $\vec{s}=\left(s_{1}, \ldots, s_{m}\right) \in G^{m}$ получен из $\vec{t}$ с помощью замены Гурвища $R_{k}$ (либо $\vec{t}$ получен из $\vec{s}$ с помощью замены Гурвица $R_{k}^{-1}$ ), если

$$
\begin{aligned}
s_{i} & =t_{i} \quad \text { для } i \neq k, k+1, \\
s_{k} & =t_{k} t_{k+1} t_{k}^{-1}, \quad s_{k+1}=t_{k} .
\end{aligned}
$$

ОПРЕДЕЛЕНИЕ (замена Гурвица в разложении на множители). Пусть $G$ группа, $t \in G$, и пусть $t=t_{1} \cdot \ldots \cdot t_{m}=s_{1} \cdot \ldots \cdot s_{m}$ - два разложения на множители элемента $t$. Мы скажем, что $s_{1} \cdot \ldots \cdot s_{m}$ получено из $t_{1} \cdot \ldots \cdot t_{m}$ с помощью замены Гурвища $R_{k}$, если $\left(s_{1}, \ldots, s_{m}\right)$ получено из $\left(t_{1}, \ldots, t_{m}\right)$ с помощью замены Гурвища $R_{k}$.

ОПРЕДЕЛЕНИЕ (әурвиц-эквивалентные разложения на множители). Два разложения на множители гурвищ-эквивалентны, если одно из них может быть получено из другого с помощью конечного числа замен Гурвица.

Мы начнем изучение этого отношения эквивалентности с утверждения о том, что брэйд-монодромия и замены Гурвица коммутируют друг с другом.

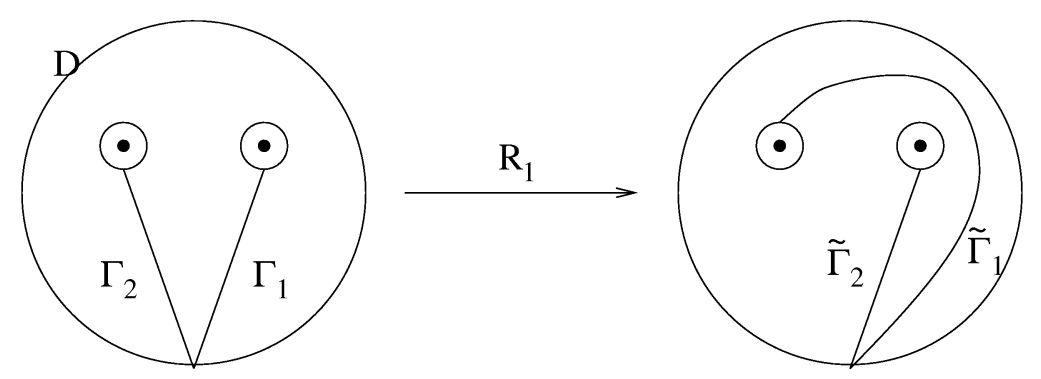

Рис. 4.1

ЛЕмма 4.1 (см. доказательство в [6, гл. II]). Пусть $D, K, u$ определень, как и выше.

a) Если $\Gamma_{1}, \ldots, \Gamma_{n}$ является $g$-базисом в $\pi_{1}(D \backslash K, u)$, то әлемент $\Gamma_{1} \cdot \ldots \cdot \Gamma_{n}$ представляется петлей $\partial D$ (взятой с положительной ориентацией).

б) $Е с л и\left\{\Gamma_{i}^{\prime}\right\}$ и $\left\{\Gamma_{i}\right\}$ - два g-базиса в $\pi_{1}(D \backslash K, u)$, то каждый әлемент $\Gamma_{i}^{\prime}$ сопряжен некоторому $\Gamma_{j_{i}}$. 
в) Применяя замену Гурвица к g-базису, мы снова получим некоторый g-базис (см. рис. 4.1).

г) Любъе два g-базиса могут быть получены один из другого с помощью конечного числа замен Гурвица.

ПРЕДЛОЖЕНИЕ 4.1. Пусть $B$ - кривая в $\mathbb{C}^{2}, u$ nусть $N, E, D, \pi, u$ onpeделены, как и выше. Пусть ч - брәйд-монодромия кривой $B$ относительно $E \times D, \pi, u$. Положим $n=\# N$. Следующая диаграмма

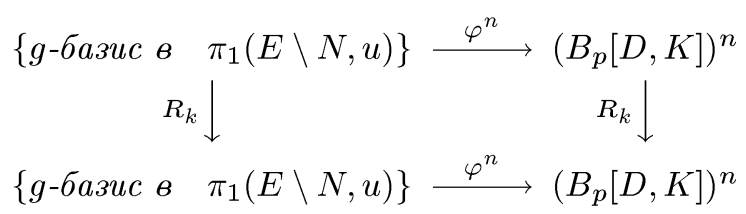

является коммутативной, где $R_{k}-k$-я замена Гурвица.

ДоКАЗАТЕЛЬСТВо следует немедленно из определений и из соотношения

$$
\varphi\left(\delta_{k} \delta_{k+1} \delta_{k}^{-1}\right)=\varphi\left(\delta_{k}\right) \varphi\left(\delta_{k+1}\right)\left(\varphi\left(\delta_{k}\right)\right)^{-1}
$$

Различные брэйд-монодромные разложения на множители, связанные с кривой $B$ и происходяшие из различных $g$-базисов, гурвищ-эквивалентны друг другу:

Лемма 4.2. Пусть $B$ - кривая, $L, \pi, E, D, N, u, K$ определены как

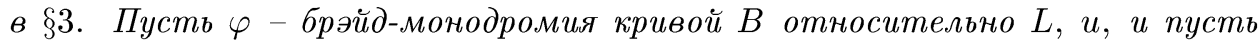
$\Delta_{p}^{2}[D, K]=\prod_{i} \varphi\left(\delta_{i}\right)$ u $\Delta_{p}^{2}[D, K]=\prod_{i} \varphi\left(\delta_{i}^{\prime}\right)$ - два брэйд-монодромных разложения на множители әлемента $\Delta_{p}^{2}[D, K]$, соответствующие $\varphi$ и двум $g$-базисам группы $\pi_{1}(E \backslash N, u)$. Тогда эти два брэйд-монодромных разложения являются гурвиц-әквивалентными.

ДоказАтЕльство. Два $g$-базиса $\left\{\delta_{i}\right\}$ и $\left\{\delta_{i}^{\prime}\right\}$ групшы $\pi_{1}(E \backslash N, u)$ могут быть получены друг из друга с помощью конечного числа замен Гурвица (лемма 4.1). По предложению 4.1 та же самая последовательность замен Гурвица преобразует $\left\{\varphi\left(\delta_{i}\right)\right\}$ в $\left\{\varphi\left(\delta_{i}^{\prime}\right)\right\}$. Таким образом, два разложения на множители $\prod_{i} \varphi\left(\delta_{i}\right)$ и $\prod_{i} \varphi\left(\delta_{i}^{\prime}\right)$ являются гурвищ-эквивалентными.

ЛЕмма 4.3. В обозначениях предыдущей леммь, если $\prod_{i} Z_{i}$ гурвиц-эквивалентно произведению $\prod \varphi\left(\delta_{i}\right)$, существует g-базис $\left\{\delta_{i}^{\prime}\right\}$ в $\pi_{1}(E \backslash N, u)$ такой, что $Z_{i}=\varphi\left(\delta_{i}^{\prime}\right)$.

ДокАЗАТЕльство. Пусть $\varepsilon$ - последовательность замен Гурвица, преобразующая $\varphi\left(\delta_{i}\right)$ в $\left\{Z_{i}\right\}$. Применяя $\varepsilon \mathrm{k}\left\{\delta_{i}\right\}$, получим некоторый $g$-базис $\left\{\delta_{i}^{\prime}\right\}$. По предложению $4.1 \varphi\left(\delta_{i}^{\prime}\right)=Z_{i}$.

Собирая вместе изложенные выше результаты, получаем следующую теорему.

TеОрема 4.1. Пусть $B$ - проективная кривая в $\mathbb{C P}^{2}$, а $\varphi: \pi_{1}(E \backslash N, u) \rightarrow$ $B_{p}[D, K]-$ связанная $с$ ней брәйд-монодромия. Множсество всех связанных $c$ В брәйд-монодромных разложсений на множители әлемента $\Delta_{p}^{2}[D, K]$ (вида

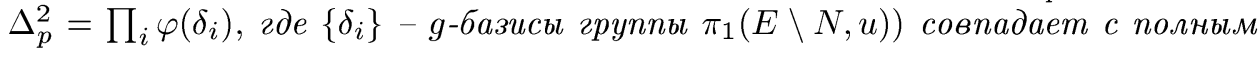


классом эквивалентности (по отношению замен Гурвица и сопряжения) разложсений на множители әлемента $\Delta_{p}^{2}$ в $B_{p}^{+}$.

Пусть $B$ - алгебраическая кривая в $\mathbb{C}^{2}$, и пусть $\varphi: \pi_{1}(E \backslash N, u) \rightarrow B_{p}[D, K]-$ связанная с ней брэйд-монодромия, определяемая брэйд-монодромным разложением на множители элемента $\Delta_{p}^{2}=\prod_{i} \varphi\left(\delta_{i}\right)$, где $\left\{\delta_{i}\right\}$ - некоторьй $g$-базис группы $\pi_{1}(E \backslash N, u)$. Действуя на $(D, K)$ диффеоморфизмом $\beta$, мы получим новое брэйд-монодромное разложение $\Delta_{p}^{2}=\prod_{i} \beta^{-1} \varphi\left(\delta_{i}\right) \beta$, связанное с кривой $B$.

ОПРЕДЕЛЕНИЕ (mun брәйд-монодромного разложения). Два брэйд-монодромных разложения на множители называются эквивалентными по отношению замен Гурвица и сопряжения, если одно из них может быть получено из другого в процессе конечного числа замен Гурвица с последующим одновременным сопряжением на некоторый элемент $\beta \in B_{p}$. Два брэйд-монодромных разложения принадлежат одному и тому же типу брәйд-разложсения, если они эквивалентны по отношению замен Гурвица и сопряжения.

\section{§5. Централизатор кратных полуповоротов}

В этом параграфе мы приведем описание образующих централизатора кратного полуповорота в группе кос, которое будет использовано в доказательстве теоремы 1. Мы воспользуемся теми же обозначениями, что и в $§ 1$.

ТЕОРема 5.1. Пусть $\left(H_{1}, \ldots, H_{p-1}\right)$ - остов группы кос $B_{p}[D, K]$, заданный путями $\left\{\sigma_{i}\right\}_{i=1}^{p-1}$, и пусть $X=H_{1}^{\nu}, \quad \nu \in \mathbb{N}$. Тогда иентрализатор $C(X)$ әлемента $X$ в $B_{p}[D, K]$ порождается әлементами $\Delta_{1, j}^{2}, j=3, \ldots, p, u H_{j}$, $j=1,3, \ldots, p-1$.

ДокАЗАТЕльство. Аналогичный результат был доказан (с использованием других методов) в [8, утверждение III.2.3]. Обозначим $C_{1}(X)$ подгруппу в $B_{p}[D, K]$, порожденную элементами $H_{1}, \Delta_{1, j}^{2}, j=3, \ldots, p$, и множеством всех полуповоротов $H(\sigma)$, заданных простыми путями $\sigma$ с началом и концом в $K$, не пересекающимися с $\sigma_{1}$, и $C_{2}(X)$ подгруппу, порожденную элементами $\Delta_{1, j}^{2}, j=3, \ldots, p$, и $H_{j}, j=1,3, \ldots, p-1$. Очевидно, $C_{2}(X) \subset C_{1}(X) \subset C(X)$ и нам надо доказать обратные включения.

Так как $C\left(X^{2}\right) \subset C(X)$, то теорему 5.1 достаточно доказать для четных $\nu$.

Не ограничивая обшности, мы можем считать, что

$$
\begin{aligned}
& D=\left\{\left(v_{1}, v_{2}\right) \in \mathbb{R}^{2} \mid v_{1}^{2}+v_{2}^{2} \leqslant p^{2}\right\}, \quad K=\{(0,0), \ldots,(p-1,0)\}, \\
& \sigma_{i}=[i-1, i]=\left\{\left(v_{1}, v_{2}\right) \in \mathbb{R}^{2} \mid i-1 \leqslant v_{1} \leqslant i, v_{2}=0\right\} .
\end{aligned}
$$

Обозначим $\sigma_{0}=[-p, 0]=\left\{\left(v_{1}, v_{2}\right) \in \mathbb{R}^{2} \mid-p \leqslant v_{1} \leqslant 0, v_{2}=0\right\}$ и $\sigma_{p}=[p-$ $1, p]=\left\{\left(v_{1}, v_{2}\right) \in \mathbb{R}^{2} \mid p-1 \leqslant v_{1} \leqslant p, v_{2}=0\right\}$. Выберем точку $u_{0} \in \partial D$ так, чтобы $u_{0}$ не лежала на прямых $\left\{v_{1}=i\right\}, i=0, \ldots, p-1$, и $\left\{v_{2}=0\right\}$. Рассмотрим элемент $\gamma \in \pi_{1}\left(D \backslash K, u_{0}\right), \gamma:[0,1] \rightarrow D \backslash K, \gamma(0)=\gamma(1)=u_{0}$. Пошевелив слегка петлю $\gamma$, мы можем считать, что она находится в общем положении с прямыми $\left\{v_{1}=i\right\}, i=0, \ldots, p-1$, и $\left\{v_{2}=0\right\}$. Выбранные координаты $\left(v_{1}, v_{2}\right)$ определяют ориентацию на $D$ и на этих прямых. Эта ориентация в каждой точке пересечения $y \in \gamma \cap L$ с ориентированной прямой $L$, находящейся с $\gamma$ в общем положении, позволяет определить индекс пересечения $(\gamma, L)_{y}$, равный \pm 1 . Пусть $t_{0}=0<$ $t_{1}<\cdots<t_{n-1}<t_{n}=1$ - последовательность значений $t \in[0,1]$, для которых 
$\gamma(t)$ принадлежит одной из упомянутых выше прямых. Сопоставим этой петле последовательность $c(\gamma)=\left(a_{0}, \ldots, a_{n}\right)$ (код петли $\left.\gamma\right)$, состоящую из символов $a_{i} \in\left\{u_{0}^{ \pm 1}, o_{0}^{ \pm 1}, \ldots, o_{p}^{ \pm 1}, h_{0}^{ \pm 1}, \ldots, h_{p-1}^{ \pm 1}, l_{0}^{ \pm 1}, \ldots, l_{p-1}^{ \pm 1}\right\}$, по следующим правилам:

1) $a_{0}=u_{0}$ и $a_{n}=u_{0}^{-1}$;

2) $a_{i}=o_{j}^{ \pm 1}$, если $\gamma\left(t_{i}\right) \in \sigma_{j}$ и степень совпадает с $\left(\gamma, \sigma_{j}\right)_{\gamma\left(t_{i}\right)}$;

3) $a_{i}=h_{j}^{ \pm 1}$, если $\gamma\left(t_{i}\right) \in L_{j}$, где $L_{j}=\left\{v_{1}=j, v_{2}>0\right\}$, и степень совпадает с $\left(\gamma_{2}, L_{j}\right)_{\gamma\left(t_{i}\right)}$

4) $a_{i}=l_{j}^{ \pm 1}$, если $\gamma\left(t_{i}\right) \in L_{j}$, где $L_{j}=\left\{v_{1}=j, v_{2}<0\right\}$, и степень совпадает с $\left(\gamma, L_{j}\right)_{\gamma\left(t_{i}\right)}$.

Код $c(\gamma)=\left(a_{0}, \ldots, a_{n}\right)$ называется приведенны.м, если $a_{i} \neq a_{i+1}^{-1}$ для любого $i$. Каждому коду $c(\gamma)=\left(a_{0}, \ldots, a_{n}\right)$ соответствует приведенный код $c_{r}(\gamma)$, полученный из $\left(a_{0}, \ldots, a_{n}\right)$ после удаления всех пар $a_{i}, a_{i+1}$ вида $a_{i}=a_{i+1}^{-1}$.

Назовем число $l_{c}(\gamma)=|c(\gamma)|=n$ длиной кода $c(\gamma)=\left(a_{0}, \ldots, a_{n}\right)$.

Аналогично, каждому пути $\sigma$, соединяющему точки $q_{i}$ и $q_{j}$ в $D \backslash\left(K \backslash\left\{q_{i}, q_{j}\right\}\right)$ можно сопоставить код $c(\sigma)$, добавив к определенным выше символам символы $q_{l}^{ \pm}$, $l=1, \ldots p$, и определить понятие приведенного кода, удовлетворяющего следующим условиям:

1) $a_{1}=q_{i}$ и $a_{n}=q_{j}^{-1}$;

2) $a_{2} \in\left\{h_{i-1}^{-1}, l_{i-1}^{-1}, h_{i+1}, l_{i+1}\right\}$;

3) $a_{n-1} \in\left\{h_{j-1}, l_{j-i}, h_{j+1}^{-1}, l_{j+1}^{-1}\right\}$;

4) $a_{l} \neq a_{l+1}^{-1}$ для любой пары $a_{l}, a_{l+1}$.

Определим знак $\operatorname{sgn}(\sigma)$ кода $c_{r}(\sigma)$, положив $\operatorname{sgn}(\sigma)=1$, если $a_{2} \in\left\{h_{i-1}^{-1}, l_{i-1}^{-1}\right\}$ и $\operatorname{sgn}(\sigma)=-1$, если $a_{2} \in\left\{h_{i+1}, l_{i+1}\right\}$.

ЛЕмма 5.1. Петли $\gamma_{0}$ и $\gamma_{1}$ (соответственно пути $\sigma^{\prime}$ и $\sigma^{\prime \prime}$ ) гомотопны в $D \backslash K$ тогда и только тогда, когда $c_{r}\left(\gamma_{1}\right)=c_{r}\left(\gamma_{2}\right)$ (соответственно $c_{r}\left(\sigma^{\prime}\right)=$ $\left.c_{r}\left(\sigma^{\prime \prime}\right)\right)$.

ДокАЗАТЕЛЬСтво. Сопоставим каждому коду $c(\gamma)=\left(a_{0}, \ldots, a_{n}\right)$ элемент $a_{0} \cdot \ldots \cdot a_{n}$ свободной группы $F$, порожденной буквами

$$
u_{0}, o_{0}, \ldots, o_{p}, h_{0}, \ldots, h_{p-1}, l_{0}, \ldots, l_{p-1}, q_{1}, \ldots, q_{p} .
$$

Очевидно, приведенному коду соответствует приведенное слово в $F$. Хорошо известно, что для каждого элемента из $F$ однозначно определено приведенное слово, представляющее этот элемент. Следовательно, для каждой петли однозначно определен приведенный код этой петли.

Если $\gamma_{0}$ и $\gamma_{1}$ гомотопны в $D \backslash K$, то, очевидно, существует такая гомотопия $\gamma_{s}$, что для почти всех $s \in[0,1]$, кроме конечного числа значений $s \in\left\{s_{1}, \ldots, s_{k}\right\}$, петли $\gamma_{s}$ находятся в общем положении с прямыми $\left\{v_{1}=i\right\}, i=0, \ldots, p-1$, и $\left\{v_{2}=0\right\}$, а для $s \in\left\{s_{1}, \ldots, s_{k}\right\}$ петли $\gamma_{s}$ касаются одной из прямых $\left\{v_{1}=\right.$ $i\}, i=0, \ldots, p-1$, и $\left\{v_{2}=0\right\}$ в одной из точек пересечения и трансверсально пересекаются с этими прямыми в остальных точках пересечения. Отсюда легко видеть, что приведенные коды гомотопных петель равны. Обратное утверждение, что если приведенные коды петель $\gamma_{0}$ и $\gamma_{1}$ равны, то $\gamma_{0}$ и $\gamma_{1}$ гомотопны, является очевидным.

Случай двух путей разбирается аналогично.

Покажем вначале, что $C_{1}(X)=C(X)$. Рассмотрим произвольный элемент $G \in C(X)$. 
ЛЕмма 5.2. Пусть диффеоморфизм $h$ является представителем әлемента $G \in C(X)$. Тогда простой путь $\sigma=h\left(\sigma_{1}\right)$ и $\sigma_{1}$ (рассматриваемые как неориентированные пути) гомотопны в $(D, K)$ (гомотопия, оставляющая на месте точки из $K$ ).

ДокАЗАТЕЛЬСТво. Имеем

$$
X=G^{-1} X G=\left(G^{-1} H\left(\sigma_{1}\right) G\right)^{\nu}=H\left(h\left(\sigma_{1}\right)\right)^{\nu}=H(\sigma)^{\nu}
$$

т. е. $X=H_{1}^{\nu}$ может быть представлен как $\nu$-я степень полуповорота, заданного путем $\sigma$. Поэтому лемма 5.2 следует из следующей леммы.

ЛЕмма 5.3. Пусть кратные полные повороты $H\left(h\left(\sigma_{1}\right)\right)^{\nu}$ u $H(\sigma)^{\nu}$ nредставляют один и тот же элемент группь $B_{p}[D, K]$, где $\sigma$-некоторый простой путь. Тогда $\sigma$ и $\sigma_{1}$ (рассматриваемые как неориентированные пути) гомотопны в $(D, K)$ (гомотопия, оставляющая на месте точки из $K$ ).

ДоказАтельство. Рассмотрим приведенный код $c_{r}(\sigma)=\left(q_{i}, a_{2}, \ldots, a_{n-1} q_{j}^{-1}\right)$. Если длина кода $\left|c_{r}(\sigma)\right|=2$, то $\sigma$ гомотопен некоторому пути $\sigma_{l}$, принадлежащему выбранному остову группы кос. Поэтому из равенства $H\left(h\left(\sigma_{1}\right)\right)^{\nu}=H\left(\sigma_{l}\right)^{\nu}$ следует $l=1$.

Покажем, что $\left|c_{r}(\sigma)\right|<3$. Действительно, пусть $\left|c_{r}(\sigma)\right| \geqslant 3$. Тогда для некоторого $s \neq 0,1$ среди элементов $a_{l}, l=1, \ldots, n$, входяших в приведенный код $c_{r}(\sigma)$, найдется элемент $a_{l_{0}} \in\left\{h_{s}^{ \pm 1}, l_{s}^{ \pm 1}\right\}$. Пусть $a_{l_{0}}=h_{s}^{\varepsilon}, \varepsilon= \pm 1$ (случай $a_{l_{0}}=l_{s}^{\varepsilon}$ разбирается аналогично, а потому он будет опущен). Выберем точку $u_{0}$ так, что $s-1<v_{1}\left(u_{0}\right)<s, v_{2}\left(u_{0}\right)>0$ и рассмотрим петлю $\gamma \in \pi_{1}\left(D \backslash K, u_{0}\right)$, приведенный код которой $c_{r}(\gamma)=\left(u_{0}, o_{s-1}, l_{s}, o_{s}^{-1}, h_{s}^{-1}, u_{0}^{-1}\right)(\gamma$ совпадает с $(p-$ $s)$-й образующей некоторого $g$-базиса). Тогда $(\gamma) H\left(\sigma_{1}\right)^{\nu}=\gamma$ и, следовательно, $c_{r}\left((\gamma) H\left(\sigma_{1}\right)^{\nu}\right)=c_{r}(\gamma)$, так как мы можем выбрать петлю, представляющую элемент $\gamma$, так, что эта петля не пересекается с $\sigma_{1}$. Вычислим код $c\left((\gamma) H(\sigma)^{\nu}\right)$. Для этого сопоставим парам $\left(q_{i}, a_{2}\right)$ и $\left(a_{n-1}, q_{j}^{-1}\right)$, входяшим в приведенный код $c_{r}(\sigma)$, четверку элементов $b_{(\sigma, \pm)}=\left(b_{1}, b_{2}, b_{3}, b_{4}\right)$, где

$$
\begin{gathered}
b_{(\sigma,+)}=\left\{\begin{array}{lll}
\left(o_{i-1}, l_{i}, o_{i}^{-1}, h_{i}^{-1}\right), & \text { если } a_{2}=h_{i-1}^{-1}, \\
\left(l_{i}, o_{i}^{-1}, h_{i}^{-1}, o_{i-1}\right), & \text { если } & a_{2}=l_{i-1}^{-1}, \\
\left(o_{i}^{-1}, h_{i}^{-1}, o_{i-1}, l_{i}\right), & \text { если } & a_{2}=l_{i+1}, \\
\left(h_{i}^{-1}, o_{i-1}, l_{i}, o_{i}^{-1}\right), & \text { если } & a_{2}=h_{i+1},
\end{array}\right. \\
b_{(\sigma,-)}=\left\{\begin{array}{lll}
\left(o_{j-1}, l_{j}, o_{j}^{-1}, h_{j}^{-1}\right), & \text { если } & a_{n-1}=h_{j-1}, \\
\left(l_{j}, o_{j}^{-1}, h_{j}^{-1}, o_{j-1}\right), & \text { если } & a_{n-1}=l_{j-1}, \\
\left(o_{j}^{-1}, h_{j}^{-1}, o_{j-1}, l_{j}\right), & \text { если } & a_{n-1}=l_{j+1}^{-1}, \\
\left(h_{j}^{-1}, o_{j-1}, l_{j}, o_{j}^{-1}\right), & \text { если } & a_{n-1}=h_{j+1}^{-1} .
\end{array}\right.
\end{gathered}
$$

Введем следующие обозначения:

$$
c_{1} c_{2}=\left(a_{1}^{\prime}, \ldots, a_{m_{1}}^{\prime}, a_{1}^{\prime \prime}, \ldots, a_{m_{2}}^{\prime \prime}\right)
$$

для $c_{1}=\left(a_{1}^{\prime}, \ldots, a_{m_{1}}^{\prime}\right)$ и $c_{2}=\left(a_{1}^{\prime \prime}, \ldots, a_{m_{2}}^{\prime \prime}\right)$ и и $c_{1}^{-1}=\left(a_{m_{1}}^{\prime-1}, \ldots, a_{1}^{\prime-1}\right)$. 
Положим

$$
\begin{aligned}
c_{r}(\sigma)_{(k+)} & =\left(a_{k+1}, \ldots, a_{n-1}\right), \quad c_{r}(\sigma)_{(k-)}=\left(a_{2}, \ldots, a_{k}\right), \\
r_{\sigma} & =\left(a_{2}, \ldots, a_{n-1}\right) b_{(\sigma,-)}\left(a_{2}, \ldots, a_{n-1}\right)^{-1} b_{(\sigma,+)}, \\
r_{\sigma, k} & =c_{r}(\sigma)_{(k+)} b_{(\sigma,-)} c_{r}(\sigma)_{(k+)}^{-1} c_{r}(\sigma)_{(k-)}^{-1} b_{(\sigma,+)} c_{r}(\sigma)_{(k-)}, \\
R_{1}(\sigma, k) & =\left[\left(c_{r}(\sigma)_{(k+)} b_{(\sigma,-)} c_{r}(\sigma)_{(k+)}^{-1}\right),\left(c_{r}(\sigma)_{(k-)}^{-1} b_{(\sigma,+)} c_{r}(\sigma)_{(k-)}\right)\right]
\end{aligned}
$$

и $R_{\nu}(\sigma, k)=r_{\sigma, k}^{\mu-1} R_{1}(\sigma, k) r_{\sigma, k}^{1-\mu}$, где $\nu=2 \mu, c_{r}(\sigma)=\left(a_{1}, a_{2}, \ldots, a_{n-1}, a_{n}\right)$, $\left[c_{1}, c_{2}\right]=c_{1} c_{2} c_{1}^{-1} c_{2}^{-1}$ и каждый код $r_{\sigma, k}$ может быть получен из $r_{\sigma}$ циклической перестановкой символов. Очевидно, $r_{\sigma, k}$ и $R_{\nu}(\sigma, k)$ являются приведенными кодами.

Не ограничивая общности, мы можем выбрать в качестве $\sigma$ и $\gamma$ такие пути, что $c(\sigma)=c_{r}(\sigma)$, а $\gamma=\delta \circ h_{s} \circ C_{s} \circ h_{s}^{-1} \circ \delta^{-1}$, где $C_{s}$ - окружность малого радиуса с центром в $q_{s}, h_{s}$ - путь вдоль прямой $v_{1}=s$ и $\delta$-кратчайший путь вдоль $\partial D$ от $u_{0}$ до пересечения $\partial D$ с лучем $\left\{v_{1}=s, v_{2}>0\right\}$. Пусть $w_{1}>w_{2}>\cdots>w_{m}>0-$ набор значений координаты $v_{2}$ точек пересечения луча $\left\{v_{1}=s, v_{2}>0\right\}$ с $\sigma$, и пусть $k_{1}, k_{2}, \ldots, k_{m}$ - соответствуюшие этим значениям номера элементов $a_{k_{l}}$, входяшие в код $c_{r}(\sigma)$ и равные по определению $h_{s}^{\varepsilon_{k_{l}}}, \varepsilon_{k_{l}}= \pm 1$. Тогда код

$$
\begin{aligned}
c\left((\gamma) H(\sigma)^{\nu}\right)= & \left(u_{0}, \prod_{l=1}^{m} R_{\nu}\left(\sigma, k_{l}\right)^{-\operatorname{sgn}(\sigma) \varepsilon_{k_{l}}, o_{s-1},}\right. \\
& \left.l_{s}, o_{s}^{-1}, h_{s}^{-1},\left(\prod_{l=1}^{m} R_{\nu}\left(\sigma, k_{l}\right)^{-\operatorname{sgn}(\sigma) \varepsilon_{k_{l}}}\right)^{-1}, u_{0}^{-1}\right),
\end{aligned}
$$

если $q_{s}$ не является началом пути $\sigma$. Если $q_{s}$ является началом пути $\sigma$, то код $c\left((\gamma) H(\sigma)^{\nu}\right)$ равен

$$
\left(u_{0}, \prod_{l=1}^{m-1} R_{\nu}\left(\sigma, k_{l}\right)^{-\operatorname{sgn}(\sigma) \varepsilon_{k_{l}}}, \delta_{0}(\sigma),\left(\prod_{l=1}^{m-1} R_{\nu}\left(\sigma, k_{l}\right)^{-\operatorname{sgn}(\sigma) \varepsilon_{k_{l}}}\right)^{-1}, u_{0}^{-1}\right)
$$

в случае $a_{2}=h_{s-1}^{-1}$, где $\delta_{0}(\sigma)=r_{\sigma}^{\mu-1} \delta_{0}^{\prime}(\sigma) r_{\sigma}^{1-\mu}$ и

$$
\begin{aligned}
\delta_{0}^{\prime}(\sigma)= & \left(a_{2}, \ldots, a_{n-1}\right) b_{(\sigma,-)}\left(a_{2}, \ldots, a_{n-1}\right)^{-1} \\
& \times b_{(\sigma,+)}\left(a_{2}, \ldots, a_{n-1}\right) b_{(\sigma,-)}^{-1}\left(a_{2}, \ldots, a_{n-1}\right)^{-1},
\end{aligned}
$$

и $c\left((\gamma) H(\sigma)^{\nu}\right)$ равен

$$
\left(u_{0}, \prod_{l=1}^{m} R_{\nu}\left(\sigma, k_{l}\right)^{-\operatorname{sgn}(\sigma) \varepsilon_{k_{l}}}, \delta(\sigma),\left(\prod_{l=1}^{m} R_{\nu}\left(\sigma, k_{l}\right)^{-\operatorname{sgn}(\sigma) \varepsilon_{k_{l}}}\right)^{-1}, u_{0}^{-1}\right)
$$

в случае $a_{2} \neq h_{s-1}^{-1}$, где

$$
\delta=\left\{\begin{array}{lll}
\left(o_{s-1}, \delta_{0}(\sigma), o_{s-1}^{-1}\right), & \text { если } a_{2}=l_{s-1}^{-1}, \\
\left(o_{s-1}, l_{s}, \delta_{0}(\sigma), l_{s}^{-1}, o_{s-1}^{-1}\right), & \text { если } a_{2}=l_{s+1}, \\
\left(o_{s-1}, l_{s}, o_{s}^{-1}, \delta_{0}(\sigma), o_{s}, l_{s}^{-1}, o_{s-1}^{-1}\right), & \text { если } a_{2}=h_{s+1} .
\end{array}\right.
$$


Не ограничивая общности, мы можем считать, что $\nu \gg 2$. Кроме того, так как $c_{r}(\sigma)$ является приведенным кодом, легко видеть, что $c_{r}\left(r_{\sigma, k_{i}} r_{\sigma, k_{j}}^{-1}\right) \neq \varnothing$ при $k_{i} \neq k_{j}$. Отсюда следует, что $c_{r}\left((\gamma) H(\sigma)^{\nu}\right) \neq c_{r}(\gamma)=\left(u_{0}, o_{s-1}, l_{s}, o_{s}^{-1}, h_{s}^{-1}, u_{0}^{-1}\right)$.

Поскольку диффеоморфизм $h$, представляюший элемент $G \in C(X) \subset B_{p}[D, K]$, определен с точностью до изотопии, согласно лемме 5.3 мы можем считать, что $h\left(\sigma_{1}\right)=\sigma_{1}, h\left(q_{i}\right)=q_{i}$ для $i=1,2$.

Покажем, что существует такой диффеоморфизм $g_{2}$, представляюший элемент $G_{2} \in C_{1}(X)$, что $g_{2} \circ h$ оставляет неподвижными пути $\sigma_{1}$ и $\sigma_{2}$. Действительно, рассмотрим путь $h\left(\sigma_{2}\right)$. Точка $q_{2}$ является началом этого пути и некоторая точка $q_{r} \neq q_{1}, q_{2}$ является его концом. Пусть $s:[0,1] \rightarrow D$ - некоторая параметризация этого пути такая, что $s(0)=q_{r}$. Рассмотрим приведенный код $c_{r}\left(h\left(\sigma_{2}\right)\right)=\left(a_{1}, \ldots, a_{n}\right)$ пути $h\left(\sigma_{2}\right), a_{1}=q_{r}, a_{n}=q_{2}$. Пусть для некоторого $i$ символ $a_{i}$ равен $o_{j}^{ \pm 1}$, где $1<j<p$. Выберем среди всех таких $i$ такое $i_{0}$, что $a_{i_{0}}=o_{j_{0}}^{ \pm 1}$ и выполнены следуюшие условия:

i) если $j_{0}=2$, то на $\sigma_{2}$ между $s\left(t_{i_{0}}\right)$ и $q_{3}$ нет других точек пересечения $h\left(\sigma_{2}\right)$ и $\sigma_{2}$

ii) если $j_{0}=r$, то на $\sigma_{r}$ между $q_{r-1}$ и $s\left(t_{i_{0}}\right)$ нет других точек пересечения $h\left(\sigma_{2}\right)$ и $\sigma_{r} ;$

iii) если $j_{0}=r+1$, то на $\sigma_{r+1}$ между $s\left(t_{i_{0}}\right)$ и $q_{r+1}$ нет других точек пересечения $h\left(\sigma_{2}\right)$ и $\sigma_{r+1}$;

iv) если $j_{0} \neq 2, r, r+1$, то на $\sigma_{j_{0}}$ между $q_{j_{0}-1}$ и $s\left(t_{i_{0}}\right)$ либо между $s\left(t_{i_{0}}\right)$ и $q_{j_{0}}$ нет других точек пересечения $h\left(\sigma_{2}\right)$ и $\sigma_{j_{0}}$.

Рассмотрим один из возможных случаев (остальные случаи аналогичны). Пусть, например, $j_{0} \neq 2, r, r+1$ и на $\sigma_{j_{0}}$ между $q_{j_{0}-1}$ и $s\left(t_{i_{0}}\right)$ нет других точек пересечения $h\left(\sigma_{2}\right)$ и $\sigma_{j_{0}}$. Обозначим через $\widetilde{h\left(\sigma_{2}\right)}$ путь, состоящий из части пути $h\left(\sigma_{2}\right)$ от точки $q_{r}$ (начало пути) до $s\left(t_{i_{0}}\right)$ и далее - вдоль пути $\sigma_{j_{0}}$ от точки $s\left(t_{i_{0}}\right)$ до $q_{j_{0}-1}$. Выберем гладкий, достаточно близкий к $\widetilde{h\left(\sigma_{2}\right)}$ путь $\widetilde{\sigma}$ такой, что $\widetilde{\sigma}$ соединяет точку $q_{r}$ (начало пути) с $q_{j_{0}-1}, \widehat{h\left(\sigma_{2}\right)} \cap \widetilde{\sigma}=\left\{q_{r}, q_{j_{0}-1}\right\}$ и при движении вдоль $\widetilde{\sigma}$ от точки $q_{r}$ путь $\widehat{h\left(\sigma_{2}\right)}$ остается все время справа от $\widetilde{\sigma}$. Осушествим полуповорот $H(\widetilde{\sigma}) \in C_{1}(X)$. Пусть диффеоморфизм $h_{1}$ является представителем элемента $H(\widetilde{\sigma})$. Легко видеть, что $h_{1}\left(h\left(\sigma_{2}\right)\right)$ изотопен пути, имеюшему код $c\left(h_{1} \circ h\right)=\left(\widetilde{a}_{0}, \ldots, \widetilde{a}_{n-i_{0}-l}\right)$, где $l-$ некоторое неотрицательное число и $\widetilde{a}_{0}=q_{j_{0}-1}$, $\tilde{a}_{j}=a_{j+i_{0}+l}$.

Обозначив $h_{1} \circ h$ снова через $h$ и повторив описанную вьше процедуру, мы можем считать, что код $c\left(h\left(\sigma_{2}\right)\right)=\left(a_{0}, \ldots, a_{n}\right)$ кривой $h\left(\sigma_{2}\right)$ удовлетворяет условию: для любого $i$ символ $a_{i} \neq o_{j}^{ \pm 1}$, где $0<j<p$. В этом случае (предполагаем для определенности, что $a_{1}=l_{r+1}$ и $a_{n-1}=l_{0}$, а остальные случаи рассматриваются аналогично) $c(h)=\left(q_{r}, l_{r+1}, \ldots, l_{p-1}, o_{p}^{-1}, h_{p-1}^{-1}, \ldots, h_{0}^{-1}, o_{0}, l_{0}, q_{2}^{-1}\right)$. Непосредственно проверяется, что $\left(h\left(\sigma_{2}\right)\right) \Delta_{r, p} \circ \Delta_{3, p} \circ \Delta_{1,2}^{2}$ изотопен пути $\sigma_{2}$. Но $\Delta_{r, p}$ 。 $\Delta_{3, p} \circ \Delta_{1,2}^{2} \in C_{2}(X) \subset C_{1}(X)$. Следовательно, умножая $G$ на $\Delta_{r, p} \circ \Delta_{3, p} \circ \Delta_{1,2}^{2}$, мы можем считать, что диффеоморфизм $h$, представляющий элемент $G \in C(X)$, оставляет на месте путь $L_{1,3}$.

Последовательно повторяя вышеизложенное для $\sigma_{3}, \ldots, \sigma_{p-1}, \sigma_{0}$, мы можем считать, что диффеоморфизм $h$, представляюший элемент $G \in C(X)$, оставляет на месте путь $L_{0, p}=\sigma_{0} \cup L_{1, p}$. В этом случае, рассматривая код пути $h\left(\sigma_{p}\right)$, легко видеть, что $h\left(\sigma_{p}\right)$ изотопен в $(D, K)$ пути $\sigma_{p}$. Поэтому мы можем считать, что $h$ 
оставляет на месте все точки диаметра $L_{0, p+1}=\sigma_{0} \cup L_{1, p} \cup \sigma_{p}$. Но в этом случае $h$ является представителем единичного элемента в $B_{p}[D, K]$. Следовательно, $C_{1}(X)=C(X)$.

Чтобы доказать, что $C_{1}(X)=C_{2}(X)$, достаточно показать, что любой полуповорот $H(\sigma)$, заданный простым, не пересекаюшимся с $\sigma_{1}$ путем $\sigma$ с началом и конщом в $K$, принадлежит $C_{2}(X)$.

Заметим, что для любого $G \in B_{p}$ элемент $G^{-1} H(\sigma) G$ является полуповоротом, заданным путем $(\sigma) G$. Поэтому доказательство того, что любой такой полуповорот $H(\sigma) \in C_{2}(X)$, с применением индукции по длине кода $|c(\sigma)|$ дословно повторяет приведенные вьше рассуждения.

\section{§6. Гладкая изотопия расслоенного пространства}

Пусть $M$ - гладкое многообразие. По определению диффеоморфизм $F: M \times$ $[0,1] \rightarrow M \times[0,1]$ (или просто $F_{t}: M \rightarrow M$ ) является гладкой изотопией, если:

1) $F(M \times\{t\})=M \times\{t\}$ для всех $t \in[0,1]$;

2) $F_{0}=\left.F\right|_{M \times\{0\}}: M \times\{0\} \rightarrow M \times\{0\}$ есть тождественное отображение.

Без ограничения общности, мы будем предполагать, что изотопия $F_{t}$ удовлетворяет еще одному дополнительному условию:

3) $F_{t}=F_{0}$, если $t \leqslant \varepsilon$, и $F_{t}=F_{1}$, если $t \geqslant 1-\varepsilon$ для некоторого $\varepsilon>0$.

Действительно, вместо гладкой изотопии $F_{t}$ мы можем рассмотреть гладкую изотопию $\widetilde{F}_{t}=F_{h(t)}$, где $h:[0,1] \rightarrow[0,1]$ - такая гладкая монотонная функция, что $h(t)=0$, если $t \leqslant \varepsilon$, и $h(t)=1$, если $t \geqslant 1-\varepsilon$.

По определению композицией гладких изотопий $F_{t}^{\prime}$ и $F_{t}^{\prime \prime}$ называется гладкая изотопия $F_{t}=F_{t}^{\prime \prime} \circ F_{t}^{\prime}$ такая, что $F_{t}=F_{2 t}^{\prime}$, если $t \leqslant \frac{1}{2}$, и $F_{t}=F_{2 t-1}^{\prime \prime} \circ F_{1}^{\prime}$, если $t \geqslant \frac{1}{2}$.

Пусть $U$ - некоторое открытое множество в $M$ и $\partial U-$ его граница. Пусть $F_{t}: \bar{U} \rightarrow \bar{U}$ - гладкая изотопия. Очевидно, что если ограничение гладкой изотопии $F_{t}: \bar{U} \rightarrow \bar{U}$ на некоторую окрестность границы $\partial U$ является тождественным отображением для всех $t \in[0,1]$, то $F_{t}$ может быть продолжена до гладкой изотопии $\widetilde{F}_{t}: M \rightarrow M$ так, что $\widetilde{F}_{t \mid M \backslash U}$ является тождественным отображением для всех $t$.

Пусть $B_{1}$ и $B_{2}$ - две плоские кривые. Как и в $\S 1$, пусть $K_{i}(x)=\{y \mid(x, y) \in$ $\left.B_{i}\right\}, i=1,2, \quad\left(K_{i}(x)\right.$ - проекция на ось $y$ множества $\left.\pi^{-1}(x) \cap B_{i}\right), N_{i}=\{x \mid$ $\left.\# K_{i}(x) \lesseqgtr p\right\}$.

$$
M_{i}^{\prime}=\left\{(x, y) \in B_{i}|\pi|_{B_{i}} \text { не этально в точке }(x, y)\right\} \quad\left(\pi\left(M_{i}^{\prime}\right)=N_{i}\right) .
$$

Пусть $E_{R}$ (соответственно $D_{R}$ ) - замкнутый диск радиуса $R$ с центром в начале координат о на оси $x$ (соответственно на оси $y$ ) такой, что $M_{i}^{\prime} \subset E_{R} \times D_{R}, N_{i} \subset$ $\operatorname{Int}(E)$. Предполагам, что \# $K_{i}(o)=p$.

Для каждого $u_{i, j} \in N_{i}, j=1, \ldots, n$, выберем диск $E_{i, j}$ малого радиуса $\epsilon \ll 1$ с центром в $u_{i, j}$ и выберем простые пути $T_{i, 1}, \ldots, T_{i, n}$, соединяюшие точки $u_{i, j}$ с o так, что $\left\langle T_{i, 1}, \ldots, T_{i, n}\right\rangle$ является кустом. Для каждого $j=1, \ldots, n$ мы выбираем маленькую трубчатую окрестность $U_{i, j}$ пути $T_{i, j}$ и диск $E_{o}$ радиуса $\epsilon \ll 1 \mathrm{c}$ центром в $о$ так, что:

1) $U_{i, j_{1}} \cap U_{i, j_{2}} \subset E_{o}$ для $j_{1} \neq j_{2}$;

2) $E_{i, j} \cap E_{o}=\varnothing$ для всех $j$;

3) множество $U_{\Gamma_{i}}=\left(\bigcup_{j=1}^{n} U_{i, j}\right) \cup\left(\bigcup_{j=1}^{n} E_{i, j}\right) \cup E_{o}$ диффеоморфно диску, и его граница $\partial U_{\Gamma_{i}}$ является гладкой простой петлей. 
Такая окрестность $U_{\Gamma_{i}}$ будет назьваться трубчатой окрестностью $g$-базиса $\Gamma_{i}=\left(l\left(T_{i, 1}\right), \ldots, l\left(T_{i, n}\right)\right)$ фундаментальной группы $\pi_{1}\left(E_{R} \backslash N_{i}, o\right)$.

Следуюшие леммы хорошо известны.

Лемма 6.1. В введенных выше обозначениях пусть $N_{i} \subset E_{R}, i=1,2,-$ два конечных множества и пусть $U_{\Gamma_{i}}-$ трубчатые окрестности g-базисов $\Gamma_{i}$ группь $\pi_{1}\left(E_{R} \backslash K_{i}, o\right)$. Предположим, что \# $N_{1}=\# N_{2}$. Тогда существует гладкая изотопия $f_{t}: E_{R} \rightarrow E_{R}, t \in[0,1]$, такая, ито:

1) $f_{t}$ является тождественным отображением в некоторой окрестности гранииь $E_{R}$;

2) $f_{t}(o)=o$ для всех $t \in[0,1]$

3) $f_{1}\left(E_{1, j}\right)=E_{2, j}$ для каждого $j=1, \ldots, n$;

4) $f_{1}\left(U_{\Gamma_{1}}\right)=U_{\Gamma_{2}}$.

Лемма 6.2. Изотопия $f_{t}$ из леммы 6.1 может быть продолжена до гладкой изотопии $F_{t}: E_{R} \times \mathbb{C}^{1} \rightarrow E_{R} \times \mathbb{C}^{1}$ такой, что

1) $F_{t \mid E_{R} \times D_{R}}=f_{t} \times \mathrm{Id}$

2) $F_{t}$ - тохсдественное отображсение вне $E_{R} \times D_{2 R}$.

ЛЕмма 6.3. Пусть гладкие вещественнье функиии $\alpha(u, v), \beta(u, v)$ удовлетворяют следующим неравенствам:

$$
\begin{aligned}
& \varepsilon_{1}+\varepsilon_{2}<\alpha(u, v)<1-\varepsilon_{1}-\varepsilon_{2}, \\
& \varepsilon_{1}+\varepsilon_{2}<\beta(u, v)<1-\varepsilon_{1}-\varepsilon_{2}
\end{aligned}
$$

для всех $(u, v) \in E_{1}=\left\{\sqrt{u^{2}+v^{2}} \leqslant 1\right\}$ и для некоторых положительных $\varepsilon_{1}, \varepsilon_{2} \ll 1$. Тогда существует гладкая вещественная функиия $f_{(\alpha, \beta) t}(z, u, v)$, $(z, t, u, v) \in[0,1] \times[0,1] \times E_{1}$, удовлетворяюшая условиям:

1) $f_{t}(z, u, v)$ является монотонной функиией для каждой фиксированной точки $(t, u, v) \in[0,1] \times E_{1}$;

2) $f_{t}(z, u, v) \equiv z$, ecлu $0 \leqslant z \leqslant \varepsilon_{1}$;

3) $f_{t}(z, u, v) \equiv z$, если $1-\varepsilon_{1} \leqslant z \leqslant 1$;

4) $f_{t}(z, u, v)=z+t(\beta(u, v)-\alpha(u, v))$, ecлu $\alpha(u, v)-\varepsilon_{2} \leqslant z \leqslant \alpha(u, v)+\varepsilon_{2}$.

ПРЕДЛОЖЕНИЕ 6.1. Пусть $\mathscr{B}=\left(b_{1}(x), \ldots, b_{p}(x)\right)$ - конечный набор не пересекаюшихся друг с другом гладких сечений проекиии $\pi: E \times D_{R} \rightarrow E=\{x \in$ $\left.\mathbb{C}^{1}|| x \mid \leqslant 1\right\}$. Тогда существует гладкая изотопия $F_{t}: E \times D_{R} \rightarrow E \times D_{R}$ такая, что:

1) $F_{t}(x, y)=\left(x, F_{t, x}(y)\right)$ для всеx $t u x$

2) $F_{1}(\mathscr{B})$ является набором постоянных сечений.

Более того, если все $b_{j}(x)$ являются постоянными сечениями (равными $\left.b_{j}\right)$ над некоторой окрестностью границы диска $E$, то изотопия $F_{t}$ может быть выбрана таким образом, что:

3) $F_{t}$ является тождественным отображением в некоторой окрестности границы множества $E \times D_{R}$ для всех $t \in[0,1]$;

4) $F_{1}\left(x, b_{j}(x)\right)=\left(x, b_{j}\right)$.

ДокАЗАТЕльСтво. Предложение будет доказано, если если мы докажем следующую лемму. 
Лемма 6.4. Пусть $\mathscr{B}=\left(b_{1}(x), b_{2}, \ldots, b_{k}\right)$ - набор не пересекающихся друг $c$ другом гладких сечений проекиии $\pi: E \times D_{R} \rightarrow E=\left\{x \in \mathbb{C}^{1}|| x \mid \leqslant 1\right\}$ такой, что $b_{2}, \ldots, b_{k}$ являются постоянными сечениями. Тогда существует такая гладкая изотопия $F_{t}: E \times D_{R} \rightarrow E \times D_{R}$, ито:

1) $F_{t}(x, y)=\left(x, F_{t, x}(y)\right)$ для всеx $t u x$;

2) $F_{t}\left(x, b_{j}\right)=\left(x, b_{j}\right)$ для всеx $t u j$;

3) $F_{1}\left(x, b_{1}(x)\right)$ является постоянным сечением.

Более того, если $b_{1}(x)$ - постоянное сечение (равное $b_{1}$ ) над некоторой окрестностью границь диска $E$, то изотопия $F_{t}$ мохсет быть выбрана таким образом, что:

4) $F_{t}$ является тожсдественным отображсением над некоторой окрестностью гранищы множества $E \times D_{R} \partial \Omega_{\text {я }}$ всех $t \in[0,1]$;

5) $F_{1}\left(x, b_{j}(x)\right)=\left(x, b_{j}\right)$.

Доказательство. Рассмотрим множество $S=\left\{y \in D_{R} \mid y=b_{1}(x), x \in E\right\}$. Предположим вначале, что мы можем найти односвязную окрестность $U \subset D_{R}$ множества $S$ такую, что $b_{j} \notin U$ для $j=2, \ldots, k$. По теореме Римана сушествует комплексно-аналитическое биективное отображение $\varphi: U \rightarrow U_{1}$, где $U_{1}=\{z=$ $\left.z_{1}+i z_{2} \in \mathbb{C} \mid 0<z_{1}<1,0<z_{2}<1\right\}$. Так как $S$ компактно, то сушествуют $\varepsilon_{1}, \varepsilon_{2}>0$ такие, что:

$$
\varepsilon_{1}+\varepsilon_{2}<z_{l}<1-\varepsilon_{1}-\varepsilon_{2}, \quad l=1,2,
$$

для $z_{1}+i z_{2} \in \varphi(S)$. Мы имеем две гладкие функции $\alpha_{1}(x), \alpha_{2}(x)$ такие, что $\varphi\left(b_{1}(x)\right)=\alpha_{1}(x)+i \alpha_{2}(x)$ и $\varepsilon_{1}+\varepsilon_{2}<\alpha_{l}(x)<1-\varepsilon_{1}-\varepsilon_{2}$. По лемме 6.3 для любых двух гладких функций $\beta_{1}(x)$ и $\beta_{2}(x)$ (например, если $\beta_{1}(x)$ и $\beta_{2}(x)$ - постоянные функции такие, что $\left.\varphi\left(b_{1}\right)=\beta_{1}+i \beta_{2}\right)$, удовлетворяюших неравенствам

$$
\varepsilon_{1}+\varepsilon_{2}<\beta_{l}(x)<1-\varepsilon_{1}-\varepsilon_{2}, \quad l=1,2,
$$

существует гладкая изотопия $F_{t}: E \times U_{1} \rightarrow E \times U_{1}$, заданная

$$
\begin{aligned}
F_{t}^{*}(x) & \equiv x \\
F_{t}^{*}\left(z_{1}\right) & =f_{\left(\alpha_{1}, \beta_{1}\right) \operatorname{th}(|x|)}\left(z_{1}, u, v\right), \\
F_{t}^{*}\left(z_{2}\right) & =f_{\left(\alpha_{l}, \beta_{l}\right) \operatorname{th}(|x|)}\left(z_{2}, u, v\right),
\end{aligned}
$$

где $u+i v=x$ и $f_{\left(\alpha_{l}, \beta_{l}\right) t}\left(z_{l}, u, v\right)$ - функция, описанная в лемме 6.3, и $h(r)$ - гладкая вещественная монотонная функция такая, что $h(r)=1$, если $r<\varepsilon$, и $h(r)=0$, если $r>1-\varepsilon$. Легко видеть, что изотопия $\varphi^{*} F_{t}$ удовлетворяет условиям 1$\left.)-4\right)$ леммы 6.4.

Мы не сможем найти такую односвязную окрестность $U$ множества $S$, только если $S$ "наматьвается" вокруг одной из точек $b_{j}, j=2, \ldots, k$. Покажем, что в этом случае существует композищия изотопий, "разматывающая" $S$. Для этого зафиксируем точку $x_{0}=1 \in E$. Пусть $\gamma(t)=x_{t}$ - гладкий простой (без самопересечений) путь, соединяющий $x_{0}$ с некоторой точкой $x_{1} \in E$. Обозначим через $n_{j, \gamma}\left(x_{1}\right)$ число полных поворотов вокруг точки $b_{j}$, двигающейся вдоль пути $b_{1}(\gamma(t))$. Очевидно, $n_{j}\left(x_{1}\right)=n_{j, \gamma}\left(x_{1}\right)$ зависит только от точки $x_{1}$ и не зависит от пути $\gamma$, соединяющего $x_{0}$ с $x_{1}$, так как $E$ односвязно и $b_{1}(x)$ - гладкая функция. Обозначим

$$
E_{n_{2}, \ldots, n_{k}}=\left\{x \in E \mid n_{j}(x)=n_{j}\right\},
$$


и пусть $E_{\max }\left(\right.$ соответственно $\left.E_{\min }\right)$ - множество $E_{n_{2}^{0}, \ldots, n_{k}^{0}}$, где $n_{j}^{0}-$ локальньй максимум (соответственно минимум) чисел $n_{j}(x)$ по всем $j$. Очевидно, если $E_{\max }=$ $E_{\min }=E_{0, \ldots, 0}$, то мы можем найти односвязную окрестность $U$ множества $S$ такую, что $b_{j} \notin U$ для $j=2, \ldots, k$. Положим $b_{1}=b_{1}\left(x_{0}\right)$.

Обозначим через $\bar{l}_{j}$ прямую, проходящую через $b_{1}$ и $b_{j}$, и выберем координату $u_{j}$ на $\bar{l}_{j}$ так, что $u_{j}\left(b_{1}\right)=0$. Пусть $l_{j}=A_{j} y_{1}+B_{j} y_{2}-$ такая линейная функция, что $\bar{l}_{j}=\left\{l_{j}\left(y_{1}, y_{2}\right)=0\right\}$, где $y_{1}=\Re y$ и $y_{2}=\Im y$.

Пусть

$$
\begin{array}{r}
S_{j}=S \cap\left\{y \in \bar{l}_{j} \mid u_{j}(y)<u_{j}\left(b_{j}\right), \text { если } u_{j}\left(b_{j}\right)>0,\right. \\
\text { и } \left.u_{j}(y)>u_{j}\left(b_{j}\right), \text { если } u_{j}\left(b_{j}\right)<0\right\} .
\end{array}
$$

Обозначим $C_{j}=\left\{x \in E \mid y=b_{1}(x) \in S_{j}\right\}$. Рассмотрим множество $G=E \backslash$ $\bigcup_{j=2}^{k} C_{j}$, являющееся несвязным объединением конечного числа связных компонент. Каждые две соседние компоненты $G_{m}$ и $G_{l}$ отделяются друг от друга некоторой связной компонентой $C_{j}^{0}$ множества $C_{j}$ для одного из $j$. Если $n_{j}(m)=n_{j}(l)$, где $n_{j}(k)=n_{j}(x)$ для $x \in G_{k}$, то мы заменим $G_{m}$ и $G_{l}$ на объединение $G_{m} \cup G_{l} \cup C_{j}^{0}$.

Очевидно, сушествует такая связная компонента $G^{0}$ множества $G$ со связной границей $\partial G^{0}$ и, следовательно, $G^{0}$ является односвязной. Более того, легко видеть, что сушествует такая односвязная окрестность $U_{0}$ множества $\left\{y \in D_{R} \mid y=\right.$ $\left.b_{1}(x), x \in \bar{g}^{0}\right\}$, что $b_{j} \notin U_{0}$ для $j=2, \ldots, k$. Пусть $j_{0}$ таково, что $\partial G^{0}$ является подмножеством в $\left\{l_{j_{0}}\left(b_{1}(x)\right)=0\right\}$. В силу выбора $G^{0}$ мы можем предположить, что $l_{j_{0}}\left(b_{1}(x)\right) \geqslant 0$ для всех $x \in G^{0}$. Так как $l_{j_{0}}\left(b_{1}(x)\right)$ - гладкая функция, множество $\left\{l_{j_{0}}\left(b_{1}(x)\right)=\delta\right\}$ является гладкой кривой для достаточно близкого к нулю числа $\delta$. Следовательно, не теряя обшности, мы можем заменить $G^{0}$ на множество $\widetilde{G}^{0}$ такое, что

1) $G^{0}$ почти совпадает с $\widetilde{G}^{0}$ и содержится в $\widetilde{G}^{0}$;

2 ) граница множества $\widetilde{G}^{0}$ является подмножеством в $\left\{l_{j_{0}}\left(b_{1}(x)\right)=\delta\right\}$ для некоторого $\delta<0$ близкого к нулю;

3) замыкание множества $b_{1}\left(\widetilde{G}^{0}\right)$ содержится в $U_{0}$, где $U_{0}$ - такое односвязное открытое множество, что $b_{j} \notin U_{0}$ для $j=2, \ldots, k$.

Как и вьше, мы можем найти голоморфную координату $z=z_{1}+i z_{2}, z=\varphi(y)$, в $U_{0}$ такую, что $U_{0} \simeq\left\{0<z_{1}<1\right\} \times\left\{0<z_{1}<1\right\}$. Очевидно, для замыкания $G^{1}$ множества $\widetilde{G}^{0}$ найдутся гладкие функции $\alpha: G^{1} \rightarrow U_{0}, z=\varphi\left(b_{1}(x)\right)=\alpha_{1}(x)+$ $i \alpha_{2}(x)$, и $\beta(x)=\beta_{1}(x)+i \beta_{2}(x)$ такие, что $\beta(x)=\varphi\left(b_{1}(x)\right)$ для $x$, лежаших в окрестности границы $\partial G^{1}$, и $l_{j_{0}}(\beta(x))<0$ для всех $x \in G^{1}$. Как и выше, применяя лемму 5.2, мы можем найти гладкую изотопию $F_{t}: G^{1} \times U_{0} \rightarrow G^{1} \times U_{0}$ такую, что $F_{1}\left(x, b_{1}(x)\right)$ не пересекается с множеством $\left\{(x, y) \mid x \in G^{1}, y \in \bigcup \bar{l}_{j}\right\}$ и $F_{t}$ является тождественным отображением в окрестности границы $G^{1} \times U_{0}$. Следовательно, эта изотопия может быть продолжена до изотопии на $E \times D_{R}$. Очевидно, для образа $F_{1}\left(b_{1}(x)\right)$ сечения $b_{1}(x)$ мы можем повторить конструкцию множества $G$ и заметить, что число связных компонент множества $G$ уменьшилось. Следовательно, после конечного числа аналогичных шагов мы построим требуемую изотопию как композицию изотопий, построенных на каждом шаге.

Из изложенного вьше следует

Замечание 6.1 . Пусть $C \subset D$ - связное множество такое, что замыкание $\bar{C}$ множества $C$ и граница $\partial D$ диска $D$ имеют непустое пересечение. Предположим, 
что $n_{j}(x)=0$ для всех $x \in C$ и для всех $j=2, \ldots, k$. Тогда на каждом шаге, кроме последнего, множество $G^{0}$ может быть выбрано так, что $C \not \subset G^{0}$.

Доказательство леммы 6.4 в случае, когда $b_{1}(x)$ является постоянным сечением над некоторой окрестностью границы диска $E$, следует из замечания 6.1.

ЗАмечАнИе 6.2. Пусть $\mathscr{B}_{1}=\left(b_{1,1}(x), \ldots, b_{1, p}(x)\right)$ и $\mathscr{B}_{2}=\left(b_{2,1}(x), \ldots\right.$ $\left.\ldots, b_{2, p}(x)\right)$ - наборы не пересекающихся друг с другом гладких сечений проекции $\pi: E \times D_{R} \rightarrow E=\left\{x \in \mathbb{C}^{1}|| x \mid \leqslant 1\right\}$ такие, что для всех $j$ сечения $b_{1, j}(x)$ и $b_{2, j}(x)$ совпадают над некоторой окрестностью $U$ гранищы диска $E$. Легко видеть, что гладкие изотопии $F_{t}^{\prime}: E \times D_{R} \rightarrow E \times D_{R}$ для $\mathscr{B}_{1}$ и $F_{t}^{\prime \prime}: E \times D_{R} \rightarrow E \times D_{R}$ для $\mathscr{B}_{2}$ из предложения 6.1 могут быть выбраны таким образом, что $F_{t}^{\prime}$ и $F_{t}^{\prime \prime}$ совпадают над $U$.

Пусть $\mathscr{B}=\left(b_{1}(x), \ldots, b_{p}(x)\right)$ - набор не пересекающихся друг с другом гладких сечений проекции $\pi: E \times D_{R} \rightarrow E=\left\{x \in \mathbb{C}^{1}|| x \mid \leqslant 1\right\}$. Обозначим

$$
U_{\varepsilon}\left(b_{j}(x)\right)=\left\{(x, y) \in E \times D_{R}|| y-b_{j}(x) \mid<\varepsilon\right\}
$$

трубчатую окрестность сечения $b_{j}(x)$ и положим $U_{\varepsilon}(\mathscr{B})=\cup U_{\varepsilon}\left(b_{j}(x)\right)$. Пусть $F_{t}: E \times D_{R} \rightarrow E \times D_{R}-$ гладкая изотопия. Рассмотрим гладкое отображение $\widetilde{F}_{t}^{\prime}: U_{\varepsilon}(\mathscr{B}) \times[0,1] \rightarrow E \times D_{R} \times[0,1]$, заданное

$$
\widetilde{F}_{t}^{\prime}(x, y)=\left(x, y+\left(F_{t}\left(b_{j}(x)\right)-b_{j}(x)\right)\right.
$$

если $(x, y) \in U_{\varepsilon}\left(b_{j}(x)\right)$. Заметим, что $\widetilde{F}_{t \mid \mathscr{B}}^{\prime}=F_{t \mid \mathscr{B}}$.

Используя стандартную технику склеивания векторных полей, можно доказать, что верна следуюшая лемма.

Лемма 6.5. Пусть изотопия $F_{t}: E \times D_{R} \rightarrow E \times D_{R}$ удовлетворяет условиям предложения 6.1. Тогда для некоторого $\varepsilon_{1} \ll \varepsilon$ отображение $\widetilde{F}_{t}^{\prime}: U_{\varepsilon_{1}}(\mathscr{B}) \times$ $[0,1] \rightarrow E \times D_{R} \times[0,1]$ может бить продолжено до гладкой изотопии $\widetilde{F}_{t}: E \times D_{R} \rightarrow E \times D_{R}$, удовлетворяющей всем условиям предложения 6.1.

Лемма 6.6. Пусть $\mathscr{B}_{i}=\left(b_{i, 1}(x), \ldots, b_{i, p}(x)\right), \quad i=1,2,-$ два набора не пересекающихся друг с другом гладких сечений проекиии $\pi: E \times D_{R} \rightarrow E=$ $\left\{x \in \mathbb{C}^{1}|| x \mid \leqslant 1\right\}$. Тогда существует гладкая изотопия $F_{t}: E \times D_{R} \rightarrow E \times D_{R}$ такая, что:

1) $F_{t}(x, y)=\left(x, F_{t, x}(y)\right)$ для всех $t u x$;

2) для каждого $j$ существует такая окрестность $U_{j}$ сечения $b_{j}(x)$, что $F_{1 \mid U_{j}}$ голоморфна по $y$;

3) $F_{1}\left(\mathscr{B}_{1}\right)=\mathscr{B}_{2}$ над $E_{R_{1}} \subset E$ для некоторого $R_{1}<1$;

4) $F_{t}$ - тохсдественное отображсение в некоторой окрестности границь множества $E \times D_{R}$ для всех $t \in[0,1]$.

ДокАЗАТЕЛЬство. Из предложения 6.1 и леммы 6.5 следует существование гладкой изотопии $\widetilde{F}_{t}: E \times D_{R} \rightarrow E \times D_{R}$, удовлетворяющей условиям 1), 2) леммы 6.6 и такой, что $\widetilde{F}_{1}\left(\mathscr{B}_{1}\right)=\mathscr{B}_{2}$. Пусть $h(r)$ - такая гладкая вешественная монотонная функция, что $h(r)=1$, если $r \leqslant R_{1}<1-\varepsilon$, и $h(r)=0$, если $r \geqslant 1-\varepsilon$. Тогда $F_{t}(x, y)=\widetilde{F}_{h(|x|) t}(x, y)$ удовлетворяет всем условиям леммы 6.6. 
ЛЕмма 6.7. Пусть $\mathscr{B}_{i}=\left(b_{i, 1}(x), \ldots, b_{i, p}(x)\right), \quad i=1,2,-$ два набора не пересекающихся друг с другом гладких сечений проекиии $\pi: E \times D_{R} \rightarrow E=$ $\left\{x \in \mathbb{C}^{1} \mid x_{1}=\Re x \in[0,1], x_{2}=\Im x \in[0,1]\right\}$ maких, ито над $E_{\varepsilon}=\left\{0 \leqslant x_{1} \leqslant \varepsilon\right\} \times$ $\left\{0 \leqslant x_{2} \leqslant 1\right\} \cap\left\{1-\varepsilon \leqslant x_{1} \leqslant 1\right\} \times\left\{0 \leqslant x_{2} \leqslant 1\right\}$ сечения $\left(b_{1,1}(x), \ldots, b_{1, p}(x)\right)=$ $\left(b_{2,1}(x), \ldots, b_{2, p}(x)\right)=\left(b_{1}, \ldots, b_{p}\right), x_{1} \in[0,1]$, суть совпадающие постоянние сечения. Пусть геометрические косы $\overline{\mathscr{B}}_{1}=\left(b_{1,1}\left(x_{1}\right), \ldots, b_{1, p}\left(x_{1}\right)\right)$ и $\overline{\mathscr{B}}_{2}=$ $\left(b_{2,1}\left(x_{1}\right), \ldots, b_{2, p}\left(x_{1}\right)\right), x_{1} \in[0,1]$, являются двумя представителями одного и того же әлемента из группь кос $B_{p}$. Тогда существует гладкая изотопия $F_{t}: E \times D_{R} \rightarrow E \times D_{R}$ такая, что:

1) $F_{t}(x, y)=\left(x, F_{t, x}(y)\right)$ для всех $t u x$;

2) для каждого $j$ существует такая окрестность $U_{j}$ сечения $b_{j}(x)$, что $F_{1 \mid U_{j}}$ голоморфно по $y$

3) $F_{1}\left(\mathscr{B}_{1}\right)=\mathscr{B}_{2}$ над $\left\{0 \leqslant x_{1} \leqslant 1\right\} \times\left\{\left|x_{2}\right|<\varepsilon_{2}\right\}$ для некоторого $\varepsilon_{2}>0$;

4) $F_{1}$ - тождественное отображение в некоторой окрестности границы множества $E \times D_{R}$.

ДокАЗАТЕЛЬСТво. Согласно предложению 6.1 существует гладкая изотопия $\widetilde{F}_{t}$ такая, что $\widetilde{F}_{1}\left(b_{1, j}(x)\right)=b_{j}, j=1, \ldots, p$, являются постоянными сечениями.

Зафиксируем точку $x_{0}=(0,0)$. Как и вьше, для каждого сечения $\widetilde{F}_{1}\left(b_{2, j_{0}}(x)\right)$ мы можем определить функцию $n_{j_{0}, j}(x)$, равную числу поворотов вокруг точки $b_{j}$. Очевидно, $F_{1}\left(\overline{\mathscr{B}}_{1}\right)$ и $F_{1}\left(\overline{\mathscr{B}}_{2}\right)$ также являются представителями одного и того же элемента из $B_{p}$. Следовательно, для каждого сечения $\widetilde{F}_{1}\left(b_{2, j_{0}}(x)\right)$ число поворотов $n_{j_{0}, j}(x)=0$ для $x \in\left\{1-\varepsilon \leqslant x_{1} \leqslant 1\right\} \times\left\{0 \leqslant x_{2} \leqslant 1\right\}$. Поэтому лемма 6.7 следует из замечания 6.1 и леммы 6.6 .

\section{§7. Типы брэйд-монодромного разложения и диффеоморфизмы пар}

Рассмотрим линейную проекцию $\pi: \mathbb{C P}^{2} \rightarrow \mathbb{C P}^{1}$ с центром в $z \in \mathbb{C P}^{2}$.

ОПРЕДЕЛЕНИЕ (полуалгебраическая кривая (относительно $\pi$ )). Замкнутое множество $B \subset \mathbb{C P}^{2}, z \notin B$, называется полуалгебраической кривой относительно проекции $\pi$, если для каждой точки $x \in B$ сушествует окрестность $U \subset \mathbb{C P}^{2}$ этой точки и аналитичекие координаты $\left(z_{1}, z_{2}\right)$ в $U, x=(0,0)$, такие, что:

1) $\left.\pi\right|_{U}$ задается равенством $\pi\left(z_{1}, z_{2}\right)=z_{1}$;

2) либо $B \cap U$ является гладким сечением проекции $\left.\pi\right|_{U}$ над $\pi(U)$, либо $B \cap U$ совпадает с множеством, заданньм уравнением $f\left(z_{1}, z_{2}\right)=0$, где $f\left(z_{1}, z_{2}\right)$ - некоторая аналитическая функция.

Полуалгебраическая кривая является (обобшенной) каспидальной кривой, если в п. 2) функция $f$ совпадает с $f=z_{1}^{k}-z_{2}^{2}, k \in \mathbb{N}$. Точка $(0,0)$, в окрестности которой $B$ задается уравнением $z_{1}^{k}-z_{2}^{2}=0$, называется особой точкой полуалгебраической кривой $B$. Очевидно, любая алгебраическая кривая $B \subset \mathbb{C P}^{2}$ является полуалгебраической относительно общей проекции.

Как и в алгебраическом случае, мы можем разрешить особые точки полуалгебраической кривой $B$ с помощью композиции $\sigma$-процессов $\nu: \overline{\mathbb{P}}^{2} \rightarrow \mathbb{P}^{2}$ (точки касания кривой $B$ со слоями проекции $\pi$ разрешать не надо) и получить неособую риманову поверхность $\bar{B} \subset \overline{\mathbb{P}}^{2}$. Композиция $\left.(\pi \circ \nu)\right|_{\bar{B}}: \bar{B} \rightarrow \mathbb{P}^{1}$ позволяет ввести на $\bar{B}$ комплексную структуру так, что $\left.(\pi \circ \nu)\right|_{\bar{B}}$ будет голоморфным отображением, однако, $\left.\nu\right|_{\bar{B}}: \bar{B} \rightarrow \mathbb{P}^{2}$ не будет голоморфным, а будет только $C^{\infty}$-отображением. 
Очевидно, как и в алгебраическом случае, для любой полуалгебраической кривой можно определить брэйд-монодромию относительно $\pi$, брэйд-монодромное разложение и брэйд-монодромный тип.

Пусть $\sigma: \mathbb{F}_{1} \rightarrow \mathbb{C P}^{2}-\sigma$-процесс с центром в $z, L=\sigma^{-1}(z)$. Обозначим снова через $\pi: \mathbb{F}_{1} \rightarrow \mathbb{C P}^{1}$ композицию $\pi \circ \sigma$.

ТЕОРЕМА 7.1. Пусть две (обобщенные) каспидальные полуалгебраических кривье $B_{1}$ и $B_{2}$ имеют один и тот же брәйд-монодромный тип $\Delta\left(B_{1}\right)=$ $\Delta\left(B_{2}\right)$. Тогда существует гладкая изотопия $F_{t}: \mathbb{F}_{1} \rightarrow \mathbb{F}_{1}$ такая, что:

1) $F_{t \mid U}-$ тождественное отображение для всех $t \in[0,1]$, где $U$ - некоторая окрестность исключительной кривой $L$;

2) для каждой точки $p \in B_{1}$ существуют окрестности $U_{1}$ точки $p u$ $U_{2}=F_{1}\left(U_{1}\right)$ точки $F_{1}(p)$ с локальнылми комплекснылми координатами $\left(x_{i}, y_{i}\right)$ в $U_{i}, i=1,2$, такие, что $\left.\pi\right|_{U_{i}}$ совпадает с проекиией $\left(x_{i}, y_{i}\right) \mapsto x_{i} u F_{1}^{*}\left(y_{2}\right)=$ $\phi\left(x_{1}, y_{1}\right)$ является гладкой $u$, более того, голоморфной по $y_{1}$ комплексной функииеи;

3) для каждой особой точки $s \in B_{1}$ существует окрестность $U \subset \mathbb{F}_{1}$ точки s такая, что $F_{1 \mid U}: U \rightarrow F(U)$ является голоморфным отображением;

4) $F_{1}\left(B_{1}\right)=\left(B_{2}\right)$.

СЛЕДСТВИЕ 7.1. Пусть две (обобщеннье) каспидальные полуалгебраические кривые $B_{1}$ и $B_{2}$ имеют один и тот жсе брәйд-монодромный тип $\Delta\left(B_{1}\right)=$ $\Delta\left(B_{2}\right)$. Тогда существует диффеоморфизм пар $F:\left(\mathbb{C P}^{2}, B_{1}\right) \rightarrow\left(\mathbb{C P}^{2}, B_{2}\right)$, удовлетворяюший условиям 2) и 3) теоремы 7.1 .

ДокаЗАТЕЛЬСтво. Назовем изотопию $F_{t}: \mathbb{F}_{1} \rightarrow \mathbb{F}_{1}$ согласованной с полуалгебраической кривой $B$ (относительно проекции $\pi$ ), если $F_{t}(B)$ является полуалгебраической кривой относительно $\pi$ для любого $t \in[0,1]$. Требуемая изотопия $F_{t}$ будет получена как композиция последовательности согласованных с $B_{1}$ гладких изотопий.

Без ограничения общности мы можем предположить, что $B_{1}$ и $B_{2}$ вложены в одно и то же пространство $\mathbb{F}_{1}$ и что они являются каспидальными алгебраическими кривыми степени $р$ относительно проекции $\pi$. Зафиксируем точку $\infty \in \mathbb{C P}^{1}$ такую, что $\pi^{-1}(\infty)$ является общим слоем проекции $\pi$ относительно каждой кривой $B_{1}$ и $B_{2}$. Обозначим $\mathbb{C}^{1}=\mathbb{C P}^{1} \backslash\{\infty\}$ и $\mathbb{C}^{2}=\mathbb{F}_{1} \backslash\left(\pi^{-1}\left(\mathbb{C}^{1}\right) \cup L\right)$. Выберем координаты $(x, y)$ в $\mathbb{C}^{2}$ так, что $\pi: \mathbb{C}^{2} \rightarrow \mathbb{C}^{1}$ является проекцией на первую координату.

Как и в $\S 6$, положим $K_{i}(x)=\left\{y \mid(x, y) \in B_{i}\right\}, i=1,2\left(K_{i}(x)\right.$ - проекция на ось $y$ множества $\left.\pi^{-1}(x) \cap B_{i}\right), N_{i}=\left\{x \mid \# K_{i}(x) \lesseqgtr p\right\}, M_{i}^{\prime}=\left\{(x, y) \in B_{i}|\pi|_{B_{i}}-\right.$ не этально в $(x, y)\}\left(\pi\left(M_{i}^{\prime}\right)=N_{i}\right)$.

Пусть $E_{R}$ (соответственно $D_{R}$ ) - замкнутый диск радиуса $R$ с центром в начале координат о на оси $x$ (соответственно на оси $y$ ) такой, что $M_{i}^{\prime} \subset E_{R} \times D_{R}, N_{i} \subset$ $\operatorname{Int}\left(E_{R}\right)$, и пусть \# $K_{i}(o)=p$.

Для каждой точки $u_{i, j} \in N_{i}, j=1, \ldots, n$, выберем диск $E_{i, j}$ радиуса $\varepsilon \ll 1 \mathrm{c}$ центром в $u_{i, j}$ и выберем пути $T_{i, 1}, \ldots, T_{i, n}$, соединяюшие точки $u_{i, j}$ с $о$ так, что $\left\langle T_{i, 1}, \ldots, T_{i, n}\right\rangle$ является кустом. Для каждого $j=1, \ldots, n$ выберем трубчатую окрестность $U_{i, j}$ пути $T_{i, j}$ и диск $E_{o}$ радиуса $\varepsilon \ll 1$ с центром в $o$ так, что множество $U_{\Gamma_{i}}=\left(\bigcup_{j=1}^{n} U_{i, j}\right) \cup\left(\bigcup_{j=1}^{n} E_{i, j}\right) \cup E_{o}$ диффеоморфно диску и его граница $\partial U_{\Gamma_{i}}$ является трубчатой окрестностью $g$-базиса $\Gamma_{i}=\left(l\left(T_{i, 1}\right), \ldots, l\left(T_{i, n}\right)\right)$ фундаментальной группы $\pi_{1}\left(E_{R} \backslash N_{i}, o\right)$. 
Из лемм 6.1 и 6.2 следует, что $E_{i, j}=\left\{x \in \mathbb{C}^{1}|| x-x_{i, j} \mid<2\right\}$, где $x_{i, j}-$ координата точки $u_{i, j}$.

Не ограничивая обшности, мы можем считать, что $T_{i, j} \cap E_{i, j}$ является радиусом в $E_{i, j}$. Продолжим этот радиус до диаметра $d_{i, j}$ и обозначим через $\widetilde{T}_{i, j}$ путь, продолжаюший $T_{i, j}$ вдоль этого диаметра, $T_{i, j} \subset \widetilde{T}_{i, j}$.

Шаг I. Так как $B_{1}$ и $B_{2}$ имеют один и тот же брэйд-монодромный тип, по лемме 4.3 мы можем выбрать (и фиксировать) $g$-базисы $\Gamma_{i}, i=1,2$, такие, что соответствуюшие им брэйд-монодромные разложения на множители $\Delta\left(B_{1}\right)$ и $\Delta\left(B_{2}\right)$ эквивалентны относительно сопряжения. Пусть $U_{\Gamma_{i}}-$ трубчатая окрестность $g$-базиса $\Gamma_{i}$.

ЛЕмма 7.1. Пусть $B \subset \mathbb{F}_{1}$ - полуалгебраическая кривая, и пусть $F_{t}: \mathbb{F}_{1} \rightarrow$ $\mathbb{F}_{1}$ - согласованная с ней гладкая изотопия такая, что $F_{t}$ - тождественное отображение над дополнением $к$ некоторой окрестности $U \subset \mathbb{C P}^{1}, \quad$ o $\notin$. Тогда кривье $B$ и $F_{1}(B)$ имеют одно и то же брәйд-монодромное разложение на множители.

ДоказАТЕЛЬСТво. Имеем непрерывное семейство гомоморфизмов $\varphi_{t}: \pi_{1}(E \backslash$ $N, o) \rightarrow B_{p}\left[\mathbb{C}_{o}, K\right]$. Так как $B_{p}\left[\mathbb{C}_{o}, K\right]$ является дискретной группой, то брэйд-монодромные разложения на множители кривых $F_{t}(B)$ не зависят от $t$.

Из лемм 6.1, 6.2 и 7.1 следует, что сушествует гладкая изотопия $F_{t}: \mathbb{F}_{1} \rightarrow \mathbb{F}_{1}$, удовлетворяюшая условиям 1)-3) теоремы 7.1 и такая, что:

1) $F_{1}\left(U_{\Gamma_{1}} \times \pi^{-1}\left(U_{\Gamma_{1}}\right)\right)=U_{\Gamma_{2}} \times \pi^{-1}\left(U_{\Gamma_{2}}\right)$;

2) $F_{1}\left(N_{1} \times \pi^{-1}\left(N_{1}\right)\right)=N_{2} \times \pi^{-1}\left(N_{2}\right)$;

3 ) типы особых точек $s_{1, j} \in F_{1}\left(B_{1}\right)$ и $s_{2, j} \in B_{2}$ над точкой $u_{2, j}=u_{j} \in N_{2}=N$ совпадают;

4) $B_{1}$ и $F_{1}\left(B_{1}\right)$ имеют одно и то же (с точностью до сопряжения) брэйд-монодромное разложение на множители.

Обозначим снова через $B_{1}$ ее образ $F_{1}\left(B_{1}\right)$.

Шаг II. По лемме 6.6 сушествуют гладкие изотопии $F_{t}^{\prime}: \mathbb{F}_{1} \rightarrow \mathbb{F}_{1}$ и $F_{t}^{\prime \prime}: \mathbb{F}_{1} \rightarrow \mathbb{F}_{1}$, удовлетворяющие условиям 1$)-3$ ) теоремы 7.1 , и такие, что $F_{1}^{\prime}\left(B_{1}\right)$ совпадают с $F_{1}^{\prime \prime}\left(B_{2}\right)$ над $E_{o}$, где $E_{o}-$ диск с центром в начале координат $o \in \mathbb{C}^{1}$. Более того, мы можем предполагать, что $F_{1}^{\prime}\left(B_{1}\right)$ и $F_{1}^{\prime \prime}\left(B_{2}\right)$ являются постоянными сечениями над $E_{o}: K(u)=\left\{y_{j} \in D_{R} \mid y_{j}=2 j-1, j \in \mathbb{Z}, 0 \leqslant j \leqslant p-1\right\}$ для всех $u \in E_{o}$.

Обозначим снова через $B_{1}$ и $B_{2}$ их образы $F_{1}^{\prime}\left(B_{1}\right)$ и $F_{1}^{\prime \prime}\left(B_{2}\right)$ соответственно.

ЗАмЕчАниЕ 7.1. Изотопии $F_{t}^{\prime}$ и $F_{t}^{\prime \prime}$ меняют брэйд-монодромные разложения на множители, связанные с кривыми $B_{1}$ и $B_{2}$, на эквивалентные относительно сопряжения, но не меняют их брэйд-монодромные типы разложения.

Зафиксируем остов $\left(\sigma_{1}, \ldots, \sigma_{p-1}\right)$ группы $B_{p}\left[\mathbb{C}_{o}^{1}, K(o)\right]$,

$$
\sigma_{j}=[2 j-3,2 j-1]=\left\{y \in \mathbb{C}^{1} \mid 2 j-3 \leqslant \operatorname{Re} y \leqslant 2 j-1, \operatorname{Im} y=0\right\} .
$$

Шаг III. Пусть в множестве $K_{i}\left(u_{j}\right)=\left\{q_{i, 1}, \ldots, q_{i, p-1}\right\}$ точка $q_{i, 1}$ является особой точкой кривой $B_{i}$, где $u_{j}$ - центр диска $E_{j}=E_{1, j}=E_{2, j}$, участвуюший в определении трубчатой окрестности $g$-базиса.

Лемма 7.2. Пусть $B=\{f(x, y)=0\}$ - росток аналитической кривой в $U=E_{\varepsilon} \times D_{\varepsilon}$, и пусть $(0,0)$ - особая точка кривой $B$ кратности 2 в направлении $x=$ const $\left(\right.$ m. e. $\#\left(B \cap\left(\{u\} \times D_{\varepsilon}\right)\right)=2$ для каждой точки $\left.u \in E_{\varepsilon}\right)$. Тогда существует гладкая изотопия $F_{t}: U \rightarrow U$ такая, что: 
1) $F_{t}=\operatorname{Id}$ в окрестности граничы множества $U$ для всех $t \in[0,1]$;

2) существует $\varepsilon_{1} \ll \varepsilon$ такое, что $F_{1}(B)=\left\{y^{2}-x^{k}=0\right\}$ для каждого $x \in E_{\varepsilon_{1}}$

3) в $V=E_{\varepsilon_{1}} \times D_{\varepsilon_{1}}$ для каждого $t$ отображение $F_{t}$ является голоморфным.

ДокАЗАТЕльство. По подготовительной теореме Вейерштрасса мы можем предполагать, что $B$ задана в окрестности $U$ уравнением

$$
y^{2}+h_{1}(x) y+h_{2}(x)=0,
$$

где $h_{i}(x)$ - аналитические функции. Запишем (3) в виде

$$
\left(y+\frac{1}{2} h_{1}(x)\right)^{2}-\left(\frac{1}{4} h_{1}^{2}(x)-h_{2}(x)\right)=\left(y+g_{1}(x)\right)^{2}-x^{k} g_{2}(x)=0,
$$

где $g_{2}(0)=c=r e^{i \varphi} \neq 0$.

Пусть $F_{t}^{\prime}$ - изотопия, заданная $F_{t}^{\prime}(x, y)=\left(x, y+t h(|x|) g_{1}(x)\right)$, где $h(r)$-гладкая монотонная функция такая, что $h(r)=1$, если $r<\varepsilon_{1} \ll \varepsilon$, и $h(r)=0$, если $r>\varepsilon-\varepsilon_{1}$.

Можно показать, что гладкое отображение $\widetilde{F}_{t}^{\prime \prime}: E_{\varepsilon_{1}} \times[0,1] \rightarrow E_{\varepsilon} \times[0,1]$, заданHое

$$
\widetilde{F}_{t}^{\prime \prime}(x)=x\left((1+(r-1) t) e^{i t \varphi}+t\left(g_{2}(x)-c\right)\right)^{1 / k},
$$

может быть продолжено до гладкой изотопии $\widetilde{F}_{t}^{\prime \prime}: E_{\varepsilon} \times[0,1] \rightarrow E_{\varepsilon} \times[0,1]$ так, что $\widetilde{F}_{t}^{\prime \prime}$ является тождественным отображением в окрестности границы множества $E_{\varepsilon}$. Тогда композиция $F_{t}=F_{t}^{\prime \prime} \circ F_{t}^{\prime}$, где $F_{t}^{\prime \prime}$ задана в $U$ следуюшим образом $F_{t}^{\prime \prime}(x, y)=\left(\widetilde{F}_{t}^{\prime \prime}(x), y\right)$, удовлетворяет всем условиям леммы 7.2.

Из лемм 6.6 и 7.2 следует, что сушествуют гладкие изотопии $F_{t}^{\prime}: \mathbb{F}_{1} \rightarrow \mathbb{F}_{1}$ и $F_{t}^{\prime \prime}: \mathbb{F}_{1} \rightarrow \mathbb{F}_{1}$, удовлетворяющие условиям 1$)-3$ ) теоремы 7.1 и такие, что:

1) $F_{1}^{\prime}\left(B_{1}\right)$ и $F_{1}^{\prime \prime}\left(B_{2}\right)$ совпадают над $\bigcup_{j=1}^{n} E_{j}^{\prime}$, где $E_{j}^{\prime} \subset E_{1, j}=E_{2, j}=E_{j}-$ некоторые маленькие окрестности точек $u_{i, j}=u_{j}$;

2) применив леммы 6.1 и 6.2 , мы можем считать, что $E_{j}^{\prime}=\left\{x \in \mathbb{C}^{1}|| x-\right.$ $\left.x_{j} \mid<2\right\}$, где $x_{j}-$ координата точки $u_{j}$;

$3)$ в окрестности точки $\left(x_{j}, 0\right)$ кривые $F_{1}^{\prime}\left(B_{1}\right)$ и $F_{1}^{\prime \prime}\left(B_{2}\right)$ заданы уравнением $y^{2}=$ $\left(x-x_{j}\right)^{k}$

4) остальные $p-2$ ветви кривых $F_{1}^{\prime}\left(B_{1}\right)$ и $F_{1}^{\prime \prime}\left(B_{2}\right)$ являются постоянными сечениями над $u \in E_{j}^{\prime}$ и $K\left(u_{j}^{\prime}\right)=\left\{y_{1}=-1, \ldots, y_{j}=2 j-3, \ldots, y_{p}=2 p-3\right\}$ для $u_{j}^{\prime}=\left\{x_{j}^{\prime}=x_{j}+1\right\}$.

Обозначим снова через $B_{1}$ и $B_{2}$ образы $F_{1}^{\prime}\left(B_{1}\right)$ и $F_{1}^{\prime \prime}\left(B_{2}\right)$ соответственно и $E_{j}^{\prime}-$ через $E_{j}$. Не ограничивая обшности, мы можем считать, что $u_{j}^{\prime} \in T_{j}$. Обозначим через $u_{j}^{\prime \prime}$ точку, лежащую на диаметре $\widetilde{T}_{j} \cap E_{j}$ и симметричную точке $u_{j}^{\prime}$ относительно центра $u_{j}$. Пусть $d_{j}^{\prime}$ - часть диаметра, заключенного между $u_{j}^{\prime}$ и $u_{j}^{\prime \prime}$.

Шаг IV. Согласно лемме 7.1 и замечанию 7.1 описанные выше изотопии не изменили типы брэйд-монодромных разложений кривых $B_{1}$ и $B_{2}$.

Запишем брэйд-монодромные разложения на множители для кривых $B_{1}$ и $B_{2}$ в выбранном выше $g$-базисе $\Gamma=\Gamma_{2}$ :

$$
\begin{aligned}
\Delta^{2} & =\prod_{j=1}^{n} Q_{j}^{-1} H_{1}^{\nu_{j}} Q_{j} \quad \text { для } B_{1}, \\
\Delta^{2} & =\prod_{j=1}^{n} Q^{-1} Q_{j}^{-1} H_{1}^{\nu_{j}} Q_{j} Q \quad \text { для } B_{2} .
\end{aligned}
$$


Покажем, что в нашем случае сушествует гладкая изотопия $F_{t}: \mathbb{F}_{1} \rightarrow \mathbb{F}_{1}$, удовлетворяющая условиям 1)-3) теоремы 7.1 и такая, что:

1) $F_{t}$ - тождественное отображение над дополнением к некоторой окрестности $U_{o}$ точки $o$;

2) $F_{1}\left(B_{1}\right)=B_{2}$ над некоторой окрестностью $U_{o}^{\prime} \subset U_{o}$;

3) $F_{1}\left(B_{1}\right)$ и $B_{2}$ имеют одинаковые брэйд-монодромные разложения на множители.

Действительно, пусть $K=\left\{y_{j} \in \mathbb{C}^{1} \mid y_{j}=2 j-3, j=1,2, \ldots, p\right\}$. Полуповорот $H_{j}=H\left(\sigma_{j}\right) \in B_{p}=B_{p}\left[\mathbb{C}_{o}^{1}, K(o)\right]$ может быть представлен геометрической косой $\bar{\sigma}_{j}\left(s_{1}\right)$ в $\mathbb{C}^{1} \times[0,1]:$

$$
\begin{aligned}
\bar{\sigma}_{j, l}\left(s_{1}\right) & =l \text { для } l=1, \ldots, j-1, j+2, \ldots, p, \\
\bar{\sigma}_{j, j}\left(s_{1}\right) & =e^{\pi\left(\beta\left(s_{1}\right)+1\right) i}+2 j-2, \\
\bar{\sigma}_{j, j+1}\left(s_{1}\right) & =e^{\pi \beta\left(s_{1}\right) i}+2 j-2,
\end{aligned}
$$

где $s_{1} \in[0,1]$ и $\beta\left(s_{1}\right)$ - вещественная гладкая монотонная функция такая, что $\beta\left(s_{1}\right)=0$ для $s_{1} \in\left[0, \frac{1}{3}\right]$ и $\beta\left(s_{1}\right)=1$ для $s_{1} \geqslant \frac{2}{3}$.

Элемент $H_{j}^{-1}$ может быть представлен геометрической косой $\bar{\sigma}_{j}^{-1}\left(s_{1}\right)$ в $\mathbb{C}^{1} \times$ $[0,1]$ :

$$
\begin{aligned}
\bar{\sigma}_{j, l}^{-1}\left(s_{1}\right) & =l \text { для } l=1, \ldots, j-1, j+2, \ldots, p, \\
\bar{\sigma}_{j, j}^{-1}\left(s_{1}\right) & =e^{\pi\left(-\beta\left(s_{1}\right)+1\right) i}+2 j-2, \\
\bar{\sigma}_{j, j+1}^{-1}\left(s_{1}\right) & =e^{-\pi \beta\left(s_{1}\right) i}+2 j-2 .
\end{aligned}
$$

Произведение $Q=H_{j_{1}}^{\delta_{1}} \cdot \ldots \cdot H_{j_{k}}^{\delta_{k}}$, где $\delta_{l}= \pm 1$, может быть представлено геометрической косой $\bar{Q}\left(s_{1}\right)$ в $\mathbb{C}^{1} \times[0, k]$ :

$$
\bar{Q}_{l}\left(s_{1}\right)=\bar{\sigma}_{j_{m}, l}^{\delta_{j_{m}}}\left(s_{1}-m+1\right) \text { для } s_{1} \in[m-1, m] .
$$

Пусть $U^{\prime} \subset U_{o}$ - некоторая окрестность точки $o$, для которой существует такой диффеоморфизм $\varphi: U^{\prime} \rightarrow V=(-1,2 k+1) \times(0,2)$, что $\varphi(o)=(0,0)$. Очевидно, пути $T_{j}$, представляющие тот же куст, могут быть выбраны таким образом, что $\varphi\left(T_{j} \cap U^{\prime}\right) \subset\left\{\left(v_{1}, v_{2}\right) \in V \mid v_{1} \leqslant 0\right\}$. Пусть $\alpha(r), r \geqslant 0,-$ вешественная гладкая монотонная функция такая, что $\alpha(r)=1$ для $r \in\left[0, \frac{4}{3}\right]$ и $\alpha(r)=0$ для $r \geqslant \frac{5}{3}$. Для $\bar{Q}=\bar{\sigma}_{j_{1}}^{\delta_{1}} \cdot \ldots \cdot \bar{\sigma}_{j_{k}}^{\delta_{k}}$ рассмотрим гладкую изотопию $F_{\bar{Q}, t}=: V \times E_{R} \rightarrow V \times E_{R}$, где

$$
F_{\bar{Q}, t}=F_{2 k, \bar{\sigma}_{j_{1}}^{-\delta_{1}, t}} \circ \cdots \circ F_{k+2, \bar{\sigma}_{j_{k-1}}^{-\delta_{k-1}, t}} \circ F_{k+1, \bar{\sigma}_{j_{k}}^{-\delta_{k}, t}} \circ F_{k, \bar{\sigma}_{j_{k}, t}^{\delta_{k}}} \circ \cdots \circ F_{1, \sigma_{j_{1}}, t}^{\delta_{1}}
$$

и $F_{l, \bar{\sigma}_{j}^{\delta_{l}}, t}$ задается функциями

$$
F_{l, \bar{\sigma}_{j} \delta_{l}, t}\left(s_{1}, s_{2}, y\right)=\left(s_{1}, s_{2}, f_{l, \bar{\sigma}_{j} \delta_{l}, t}\left(s_{1}, s_{2}, y\right),\right.
$$

где

$$
f_{l, \bar{\sigma}_{j}^{\delta_{l}}, t}\left(s_{1}, s_{2}, y\right)= \begin{cases}y, & s_{1} \leqslant l-1 ; \\ 2 j-2+(y-2 j+2) & \\ \quad \times e^{i \pi \delta_{j} \alpha\left(s_{2}\right) \beta\left(s_{1}-l+1\right) \alpha(|y-2 j+2|) t}, & l-1 \leqslant s_{1} \leqslant l ; \\ 2 j-2+(y-2 j+2) & \\ \times e^{i \pi \delta_{j} \alpha\left(s_{2}\right) \alpha(|y-2 j+2|) t}, & s_{1} \geqslant l,\end{cases}
$$


если $l \leqslant k$, и

$$
f_{l, \bar{\sigma}_{j}^{\delta_{l}}, t}\left(s_{1}, s_{2}, y\right)= \begin{cases}y, & s_{1} \geqslant l ; \\ 2 j-2+(y-2 j+2) & \\ \quad \times e^{i \pi \delta_{j} \alpha\left(s_{2}\right) \beta\left(l-s_{1}\right) \alpha(|y-2 j+2|) t}, & l-1 \leqslant s_{1} \leqslant l ; \\ 2 j-2+(y-2 j+2) & \\ \quad \times e^{i \pi \delta_{j} \alpha\left(s_{2}\right) \alpha(|y-2 j+2|) t}, & s_{1} \leqslant l-1,\end{cases}
$$

если $l \geqslant k+1$. Можно проверить, что:

1) $F_{\bar{Q}, t}$ - тождественное отображение над окрестностью границы множества $V$ для всех $t$;

2) $F_{\bar{Q}, 1}(\overline{\mathscr{B}})=\bar{Q}$, где $\overline{\mathscr{B}}=\left\{b_{1}\left(x\left(s_{1}, 0\right)\right) \equiv-1, \ldots, b_{p}\left(x\left(s_{1}, 0\right)\right) \equiv 2 p-3\right\}$, $0 \leqslant s_{1} \leqslant k,-$ тривиальная геометрическая коса;

3) $F_{\bar{Q}, 1}\left(\mathscr{B}_{1}\right)=\left\{\left(s_{1}, s_{2},-1\right),\left(s_{1}, s_{2}, 1\right), \ldots,\left(s_{1}, s_{2}, 2 p-3\right)\right\}$ - постоянные сечения над $\left\{k-\frac{1}{3}<s_{1}<k+\frac{1}{3}\right\} \times\left\{0<s_{2}<2\right\}$.

Такую изотопию $F_{\bar{Q}, t}$ будем называть $\bar{Q}$-скручиванием-раскручиванием постоянных сечений с носителем $\varphi^{-1}(V)$ и с иентром $\left(V_{0}, z_{0}\right)$, где

$$
V_{0}=\varphi^{-1}\left(\left\{k-\frac{1}{3}<s_{1}<k+\frac{1}{3}\right\} \times\left\{0<s_{2}<2\right\}\right)
$$

и $z_{0}=\varphi^{-1}((k, 0))$.

Пусть $\widetilde{F}_{\bar{Q}, t}=\varphi^{*}\left(F_{\bar{Q}, t}\right)$. Обозначим снова через $B_{1}$ ее образ $\widetilde{F}_{\bar{Q}, 1}\left(B_{1}\right)$.

В обозначениях определения $g$-базиса $\Gamma$ и его трубчатой окрестности мы заменим $g$-базис $\Gamma$ на эквивалентный ему, взяв $z_{0}$ вместо $o$ и заменив каждый путь $T_{j}$ на некоторый путь с началом в точке $z_{0}$ и совпадающий с $T_{j}$ вне диска $E_{o}$. Окрестность $E_{o}$ заменим на некоторый диск $E_{z_{0}} \subset V_{0}$ с центром в $z_{0}$ и выберем новые окрестности $U_{j}$, лежашие в старых $U_{j}$. В дальнейшем точку $z_{0}$ мы снова будем обозначать через $O$.

По построению $\bar{Q}$-скручивания-раскручивания $F_{\bar{Q}, t}$ брэйд-монодромное разложение на множители, соответствуюшие кривой $B_{1}$, имеет вид

$$
\Delta^{2}=\prod_{j=1}^{n} Q^{-1} Q_{j}^{-1} H_{1}^{\nu_{j}} Q_{j} Q
$$

т. е. соответствуюшие кривым $B_{1}$ и $B_{2}$ брэйд-монодромные разложения на множители совпадают.

Шаг V. Пусть $u_{j}^{\prime}$ - выбранная на шаге III точка, $u_{j}^{\prime} \in T_{j}$, и пусть $U_{\Gamma}^{\prime} \subset U_{\Gamma}-$ некоторая трубчатая окрестность $g$-базиса $Г$ такая, что $U_{\Gamma}^{\prime} \cap U\left(\partial U_{\Gamma}\right)=\varnothing$, где $U\left(\partial U_{\Gamma}\right)$ - некоторая окрестность гранищы $\partial U_{\Gamma}$ трубчатой окрестности $U_{\Gamma}$.

Покажем, что в нашем случае существует гладкая изотопия $F_{t}: \mathbb{F}_{1} \rightarrow \mathbb{F}_{1}$, удовлетворяющая условиям 1)-3) теоремы 7.1 и такая, что:

1) $F_{t}$ является тождественным отображением над дополнением к $U_{\Gamma}$;

2) $F_{1}\left(B_{1}\right)=B_{2}$ над $U_{\Gamma}^{\prime}$.

Чтобы показать это, рассмотрим для каждого $j$ геометрические косы

$$
\overline{\mathscr{B}}_{1}=\left(b_{1, j, 1}(x), \ldots, b_{1, j, p}(x)\right) \text { и } \overline{\mathscr{B}}_{2}=\left(b_{2, j, 1}(x), \ldots, b_{2, j, p}(x)\right),
$$


где $x$ движется вдоль $T_{j}^{\prime} \subset T_{j}$ с начальной точкой $о$ и конечной точкой $u_{j}^{\prime}$. Эти геометрические косы представляют элементы $\beta_{i, j} \in B\left[\mathbb{C}_{o}, K\right], i=1,2$. Так как множители в брэйд-монодромных разложениях на множители для кривых $B_{1}$ и $B_{2}$ совпадают между собой, то

$$
\beta_{1, j}^{-1} H_{1}^{\nu_{j}} \beta_{1, j}=\beta_{2, j}^{-1} H_{1}^{\nu_{j}} \beta_{2, j}=Q_{j}^{-1} H_{1}^{\nu_{j}} Q_{j} .
$$

Следовательно, $\beta_{j}=\beta_{1, j} \beta_{2, j}^{-1} \in C\left(H_{1}\right)$. По теореме 5.1 элемент $\beta_{j}$ может быть записан в виде произведения $\beta_{j}=\mu_{j, 1} \ldots \mu_{j, k_{j}}$, где каждый $\mu_{j, i}$ совпадает либо с $H_{r}^{\delta_{j, i}}$, где $\delta_{j, i}= \pm 1$ и $r=1,3, \ldots, p$, либо с поворотом $\Delta_{1, r}^{2 \delta_{j, i}}, r=3, \ldots, p$, заданным остовом $\left(\sigma_{1}, \ldots, \sigma_{p-1}\right)$.

$\mathrm{C}$ каждым таким $\beta_{j}$ мы можем связать некоторое "скручивание-раскручивание" пространства $V_{j} \times \mathbb{C}^{1}$ аналогично тому, как это было сделано на предыдущем шаге. А именно, рассмотрим снова $V_{j}=\left(-1,2 k_{j}+1\right) \times(0,2)$ и для каждого $\mu_{j, i}$ определим гладкие изотопии $F_{j, \mu_{j, i}, t}: V_{j} \times \mathbb{C} \rightarrow V_{j} \times \mathbb{C}$, а элементу $\beta_{j}=\mu_{j, 1} \ldots \mu_{j, k_{j}}$ сопоставим композицию изотопий

$$
\begin{aligned}
F_{j, \beta_{j}, t}= & F_{j, 2 k_{j}, \mu_{j, 1}^{-1}, t} \circ \cdots \circ F_{j, k_{j}+2, \mu_{j, k_{j}-1}^{-1}, t} \\
& \circ F_{j, k_{j}+1, \mu_{j, k_{j}}^{-1}, t} \circ F_{j, k_{j}, \mu_{j, k_{j}}, t} \circ \cdots \circ F_{j, 1, \mu_{j, 1}, t}
\end{aligned}
$$

следующим образом. Если $\mu_{j, l}=H\left(\sigma_{r}\right)^{\delta_{j, l}}$, то $F_{j, l, \mu_{j, l}, t}=F_{l, \bar{\sigma}_{r}{ }_{j, l}, t}$, которая была определена на предыдушем шаге (число $k$, участвующее в определении $F_{l, \bar{\sigma}_{r}{ }_{j, l}, t}$, в нашем случае равно $k_{j}$ ). Если $\mu_{j, l}=\Delta_{1, r}^{2 \delta_{j, l}}$, то $F_{j, l, \mu_{j, l}, t}$ определяется аналогично, а именно, задается функциями

$$
F_{j, l, \mu} \delta_{j, l, t}\left(s_{1}, s_{2}, y\right)=\left(s_{1}, s_{2}, f_{j, l, \mu^{\delta}, l, t}\left(s_{1}, s_{2}, y\right)\right)
$$

где

$$
f_{j, l, \mu} \delta_{j, l, t}\left(s_{1}, s_{2}, y\right)=\left\{\begin{array}{rlrl}
y, & & s_{1} \leqslant l-1 ; \\
r-2+(y-r+2) & & \\
& \times e^{2 i \pi \delta_{j, l} \alpha\left(s_{2}\right) \beta\left(s_{1}-l+1\right) \gamma(|y-r+2|) t}, & l-1 \leqslant s_{1} \leqslant l ; \\
r- & 2+(y-r+2) & & \\
& \times e^{2 i \pi \delta_{j, l} \alpha\left(s_{2}\right) \alpha(|y-r+2|) t}, & & s_{1} \geqslant l,
\end{array}\right.
$$

если $l \leqslant k_{j}$, и

$$
f_{l, \mu_{j, l}, t}\left(s_{1}, s_{2}, y\right)=\left\{\begin{array}{rlrl}
y, & & s_{1} \geqslant l ; \\
r- & 2+(y-r+2) & \\
& \times e^{2 i \pi \delta_{j, l} \alpha\left(s_{2}\right) \beta\left(l-s_{1}\right) \gamma(|y-r+2|) t}, & l-1 \leqslant s_{1} \leqslant l ; \\
r- & 2+(y-r+2) & & \\
& \times e^{2 i \pi \delta_{j, l} \alpha\left(s_{2}\right) \gamma(|y-r+2|) t}, & & s_{1} \leqslant l-1,
\end{array}\right.
$$

если $l \geqslant k_{j}+1$, где $\alpha(s), \beta(s)$ и $\gamma(s), s \geqslant 0,-$ вешественные гладкие монотонные функции такие, что $\alpha(s)=1$ для $s \in\left[0, \frac{4}{3}\right]$ и $\alpha(s)=0$ для $s \geqslant \frac{5}{3}, \beta(s)=0$ для $s \in\left[0, \frac{1}{3}\right]$ и $\beta(s)=1$ для $s \geqslant \frac{2}{3}$, а $\gamma(s)=1$ для $s \in[0, r-1]$ и $\gamma(s)=0$ для $s \geqslant r-\frac{1}{2}$. 
Выберем в диске $E_{j}$ окрестность $W_{j}$, содержащую часть диаметра $d_{j}^{\prime}$, соединяющего точки $u_{j}^{\prime}$ и $u_{j}^{\prime \prime}$, и такую, что сушествует диффеоморфизм $\phi_{j}: W_{j} \rightarrow V_{j}$ такой, что $\phi_{j}\left(d_{j}^{\prime}\right)=\left\{\left(s_{1}, s_{2}\right) \in V_{j} \mid 0 \leqslant s_{1} \leqslant 2 k_{j}, s_{2}=0\right\}$ и $\phi_{j}\left(u_{j}\right)=(0,0)$. Этот диффеоморфизм $\phi_{j}$ и изотопия $F_{j, \beta_{j}, t}$ позволяют определить гладкую изотопию $\widetilde{F}_{j, \beta_{j}, t}=\left(\phi_{j}^{-1} \times \mathrm{Id}\right) \circ F_{j, \beta_{j}, t} \circ\left(\phi_{j} \times \mathrm{Id}\right): W_{j} \times \mathbb{C}^{1} \rightarrow W_{j} \times \mathbb{C}^{1}$, которая может быть продолжена до гладкой изотопии, тождественной вне $W_{j} \times \mathbb{C}^{1}$. Пусть $F_{t}-$ композиция построенных изотопий $\widetilde{F}_{j, \beta_{j}, t}, j=1, \ldots, n$. Обозначим снова через $B_{1}$ ее образ $F_{1}\left(B_{1}\right)$ и через $E_{j}$ - некоторый диск в $V_{0, j}$, где $V_{0, j}$ - центр “скручивания-раскручивания" $\widetilde{F}_{j, \beta_{j}, t}$, который определяется дословно, как и в шаге IV. Выберем в новой окрестности $E_{j}$ новую точку, лежащую на $T_{j}$, и обозначим ее снова через $u_{j}^{\prime}$. По построению изотопий $\widetilde{F}_{j, \beta_{j}, t}$ для каждого $j$ геометрические косы

$$
\overline{\mathscr{B}}_{1}=\left(b_{1, j, 1}(x), \ldots, b_{1, j, p}(x)\right) \text { и } \overline{\mathscr{B}}_{2}=\left(b_{2, j, 1}(x), \ldots, b_{2, j, p}(x)\right)
$$

где $x$ движется вдоль $T_{j}^{\prime} \subset T_{j}$ с начальной точкой $о$ и конечной точкой $u_{j}^{\prime}$, представляют уже один и тот же элемент в $B_{p}\left[\mathbb{C}_{o}^{1}, K\right]$. Следовательно, по лемме 6.7 существует гладкая изотопия $F_{t}: \mathbb{F}_{1} \rightarrow \mathbb{F}_{1}$, удовлетворяющая условиям 1)-3) теоремы 7.1 и такая, что:

1) $F_{t}$ является тождественным отображением над дополнением к объединению достаточно маленьких окрестностей $U_{j}$ путей $T_{j}^{\prime}$;

2) $F_{t}$ является тождественным отображением над $\left(\cup E_{j}\right) \cup E_{o}$;

3) $F_{1}\left(B_{1}\right)=B_{2}$ над некоторой трубчатой окрестностью $U_{\Gamma}^{\prime} \subset U_{\Gamma} g$-базиса $\Gamma$.

Обозначим снова через $B_{1}$ ее образ $F_{1}\left(B_{1}\right)$. Полученные $B_{1}$ и $B_{2}$ совпадают над трубчатой окрестностью $U_{\Gamma}^{\prime} g$-базиса $\Gamma$.

Шаг VI. Дополнение $\mathbb{P}^{1} \backslash U_{\Gamma}^{\prime}$ является односвязным. Пусть $U_{\infty}$ - такая односвязная окрестность множества $\mathbb{P}^{1} \backslash U_{\Gamma}^{\prime}$, что $U_{\infty}$ диффеоморфна диску и такая, что $u_{j} \notin U_{\infty}$ для всех $j=1, \ldots, \# N$. Обозначим $V=\pi^{-1}\left(U_{\infty}\right)$. Тогда $\pi: V \rightarrow U_{\infty}$ является тривиальным расслоением со слоями $\pi^{-1}(x) \simeq \mathbb{P}^{1}$ и $L \cap V$ является сечением. Положим $V_{0}=V \backslash L$ и $\mathscr{B}_{i}=V_{0} \cap B_{i}, i=1,2$. Тогда $V_{0} \simeq U_{\infty} \times \mathbb{C}^{1}$ и $\left.\pi\right|_{V_{0}}$ совпадает с проекцией на второй множитель. Легко видеть, что $\mathscr{B}_{1}$ и $\mathscr{B}_{2}$ удовлетворяют всем условиям замечания 6.2. Следовательно, существует гладкая изотопия $F_{t}$, удовлетворяющая всем условиям теоремы 7.1. 


\section{§8. Типы брэйд-монодромного разложения и диффеоморфные типы поверхностей}

В этом параграфе мы докажем теорему 2.

Пусть $F:\left(\mathbb{C P}^{2}, B_{1}\right) \rightarrow\left(\mathbb{C P}^{2}, B_{2}\right)$ - диффеоморфизм пар, удовлетворяющий условиям следствия 7.1. Этот диффеморфизм индуцирует изоморфизм

$$
F^{*}: \pi_{1}\left(\mathbb{C P}^{2} \backslash B_{2}\right) \rightarrow \pi_{1}\left(\mathbb{C P}^{2} \backslash B_{1}\right) .
$$

Из предложения 1 в [3] следует, что множество неэквивалентных общих морфизмов степени $N$ с дискриминантной кривой $B \subset \mathbb{C P}^{2}$ находится во взаимно однозначном соответствии с множеством эпиморфизмов из $\pi_{1}\left(\mathbb{C P}^{2} \backslash B\right)$ в симметрическую группу $\Sigma_{N}$, удовлетворяюших некоторым дополнительньм условиям (см. детали в [3]). Так как гипотеза Кизини выполнена для $B_{1} \subset \mathbb{C P}^{2}$, то существует единственный такой эпиморфизм из $\pi_{1}\left(\mathbb{C P}^{2} \backslash B_{1}\right)$ и он должен совпадать с эпиморфизмом $f_{1 *}: \pi_{1}\left(\mathbb{C P}^{2} \backslash B_{1}\right) \rightarrow \Sigma_{N}$, индуцированньм морфизмом $f_{1}$, где $N=\operatorname{deg} f_{1}$. Поэтому для $B_{2}$ также сушествует единственный такой эпиморфизм и он должен совпадать с $f_{1 *} \circ F^{*}=f_{2 *}: \pi_{1}\left(\mathbb{C P}^{2} \backslash B_{2}\right) \rightarrow \Sigma_{N}$. Следовательно, диффеоморфизм $F: \mathbb{C P}^{2} \backslash B_{1} \rightarrow \mathbb{C P}^{2} \backslash B_{2}$ может быть поднят до диффеоморфизма $\Psi_{0}: S_{1} \backslash f_{1}^{-1}\left(B_{1}\right) \rightarrow S_{2} \backslash f_{2}^{-1}\left(B_{2}\right)$.

В [4] изложен метод восстановления поверхности $S$ и конечного морфизма $f: S \rightarrow$ $\mathbb{C P}^{2}$, разветвленного вдоль кривой $B \subset \mathbb{C P}^{2}$, по заданному гомоморфизму $f_{*}: \pi_{1}\left(\mathbb{C P}^{2} \backslash B\right) \rightarrow \Sigma_{N}$. Этот метод основан на представлении $S$ в виде $N$ экземпляров $\mathbb{C P}^{2}$ со "стандартными разрезами", склеенных друг с другом вдоль этих разрезов (чтобы осуществить такое склеивание, используется геометрическое описание конечного представления группы $\pi_{1}\left(\mathbb{C P}^{2} \backslash B\right)$ в терминах “теней” и “экранов", описанное в [5]). Используя этот метод, легко видеть, что диффеоморфизм $\Psi_{0}$ однозначно продолжается до гомеоморфизма $\Psi: S_{1} \rightarrow S_{2}$.

Пусть $U \subset \mathbb{C P}^{2}$ - окрестность обыкновенного каспа кривой $B_{1}$ такая, что $\left.F\right|_{U}$ является голоморфным отображением. Хорошо известно, что если $f: X \rightarrow U-$ трехлистное накрытие $U=\left\{(x, y) \in \mathbb{C}^{2}|| x|<1| y \mid,<1\right\}$, разветвленное вдоль кривой, заданной уравнением $y^{2}=x^{3}$, и такое, что $f$ не является накрытием Галуа, то такое $f$ единственно. Следовательно, гомеморфизм $\Psi$ является голоморфньм (в частности, $\Psi$ гладкое отображение) в $f_{1}^{-1}(U)$. Аналогично, $\Psi$ является гладким отображением в $f_{1}^{-1}(U)$, где $U$ - окрестность ноуда кривой $B_{1}$ или точки касания кривой $B_{1}$ со слоем проекции $\pi$.

Пусть $z \in B_{1}$ - неособая точка, и пусть $U_{1} \simeq\left\{\left(x_{1}, y_{1}\right) \in \mathbb{C}^{2}|| x_{1}|<1,| y_{1} \mid<1\right\}$ окрестность точки $z$ в $\mathbb{C P}^{2}$, где $\left(x_{1}, y_{1}\right)$ - локальные голоморфные координаты в $\mathbb{C P}^{2}$ такие, что $y_{1}=0$ является локальньм уравнением кривой $B_{1}$ и $\pi$ в $U_{1}$ совпадает с $\left(x_{1}, y_{1}\right) \mapsto x_{1}$. Аналогично, пусть $U_{2}=F\left(U_{1}\right)$ - окрестность точки $F(z)$, и пусть $\left(x_{2}, y_{2}\right)$ - локальные голоморфные координаты в $U_{2}$ такие, что $y_{2}=0$ является локальным уравнением кривой $B_{2}$ и $\pi$ в $U_{2}$ совпадает с $\left(x_{2}, y_{2}\right) \mapsto x_{2}$. Имеем: $x_{2}=g_{1}\left(x_{1}\right)$ и $y_{2}=g_{2}\left(x_{1}, y_{1}\right)$, где $g_{1}$ и $g_{2}$ - гладкие функции и $g_{2}$ голоморфна по $y_{1}$. Следовательно, $g_{2}$ может быть записана в виде

$$
g_{2}\left(x_{1}, y_{1}\right)=\sum_{n=1}^{\infty} a_{n}\left(x_{1}\right) y_{1}^{n}
$$

где все $a_{n}\left(x_{1}\right)$ - гладкие функции и $a_{1}\left(x_{1}\right) \neq 0$ в $U_{1}$. 
Каждый из прообразов $f_{1}^{-1}\left(U_{1}\right)$ и $f_{2}^{-1}\left(U_{2}\right)$ состоит из $N-1$ связной компоненты $U_{1,1}, \ldots, U_{1, N-1}$ и $U_{2,1}, \ldots, U_{2, N-1}$ соответственно. Пусть $f_{1}$ (соответственно $f_{2}$ ) не разветвлен в $\bigcup_{j=2}^{N-1} U_{1, j}$ (соответственно в $\left.\bigcup_{j=2}^{N-1} U_{2, j}\right)$. Следовательно, ограничение $\Psi$ на $\bigcup_{j=2}^{N-1} U_{1, j}$ является гладким отображением. Кроме того, сушествуют локальные голоморфные координаты $\left(u_{1}, v_{1}\right)$ в $U_{1,1}\left(\right.$ соответственно $\left(u_{2}, v_{2}\right)$ в $\left.U_{2,1}\right)$ такие, что $f_{1}$ задано в $U_{1,1}$ (соответственно $f_{2}$ задано в $U_{2,1}$ ) функциями $y_{1}=u_{1}^{2}$, $x_{1}=v_{1}$ (соответственно $\left.y_{2}=u_{2}^{2}, x_{2}=v_{2}\right)$. Отсюда следует, что $\Psi$ задается функциями

$$
\begin{aligned}
& u_{2}=u_{1}\left(\sum_{n=1}^{\infty} a_{n}\left(v_{1}\right) u_{1}^{2 n-2}\right)^{\frac{1}{2}} \\
& v_{2}=v_{1}
\end{aligned}
$$

Легко видеть, что $\left(\sum_{n=1}^{\infty} a_{n}\left(v_{1}\right) u_{1}^{2 n-2}\right)^{\frac{1}{2}}$ является гладкой функцией, так как все $a_{n}\left(v_{1}\right)$ - гладкие функции и $a_{1}\left(v_{1}\right) \neq 0$.

\section{Список литературы}

1. Artin E. Theory of braids // Ann. Math. 1947. V. 48. P. 101-126.

2. Birman J. Braids, Links and Mapping Class Groups. Princeton University Press, 1975.

3. Куликов Вик. С. О гипотезе Кизини // Изв. РАН. Сер. матем. 1999. Т. 63 . №6. C. $84-116$.

4. Куликов Вик. C. Геометрическая реализация $C$-групп // Изв. РАН. Сер. матем. 1994. T. 58. № 4. C. 194-203.

5. Kulikov Vik.S. On the fundamental group of the complement of a hypersurface in $\mathbb{C}^{n}$ // Springer L.N.M. 1991. V. 1479. P. 122-130.

6. Moishezon B., Teicher M. Braid Groups, Singularities and Algebraic Surfaces. Birkhauser (to appear).

7. Moishezon B., Teicher M. Braid group techniques in complex geometry I. Line arrangements in $\mathbb{C P}^{2} / /$ Contemporary Math. 1988. V. 78. P. $425-555$.

8. Moishezon B., Teicher $M$. Braid group techniques in complex geometry V. The fundamental group of a complement of a branch curve of a Veronese generic projection // Communications in Analysis and Geometry. 1996. V. 4. № 11. P. 1-120.

Математический институт им. В. А. Стеклова РАН

E-mail: victor@olya.ips.ras.ru

Поступило в редакцию

Университет Бар-Илан, Израиль

E-mail: teicher@macs.biu.ac.il 